\title{
Evidence-based treatment of neurogenic orthostatic hypotension and related symptoms
}

\author{
Sabine Eschlböck ${ }^{1}$ - Gregor Wenning ${ }^{1}$ - Alessandra Fanciulli ${ }^{1}$ [D
}

Received: 27 July 2017/ Accepted: 18 September 2017/Published online: 22 October 2017

(C) The Author(s) 2017. This article is an open access publication

\begin{abstract}
Neurogenic orthostatic hypotension, postprandial hypotension and exercise-induced hypotension are common features of cardiovascular autonomic failure. Despite the serious impact on patient's quality of life, evidence-based guidelines for non-pharmacological and pharmacological management are lacking at present. Here, we provide a systematic review of the literature on therapeutic options for neurogenic orthostatic hypotension and related symptoms with evidence-based recommendations according to the Grading of Recommendations Assessment, Development and Evaluation (GRADE). Patient's education and non-pharmacological measures remain essential, with strong recommendation for use of abdominal binders. Based on quality of evidence and safety issues, midodrine and droxidopa reach a strong recommendation level for pharmacological treatment of neurogenic orthostatic hypotension. In selected cases, a range of alternative agents can be considered (fludrocortisone, pyridostigmine, yohimbine, atomoxetine, fluoxetine, ergot alkaloids, ephedrine, phenylpropanolamine, octreotide, indomethacin, ibuprofen, caffeine, methylphenidate and desmopressin), though recommendation strength is weak and quality of evidence is low (atomoxetine, octreotide) or very low (fludrocortisone, pyridostigmine, yohimbine, fluoxetine, ergot alkaloids, ephedrine, phenylpropanolamine, indomethacin, ibuprofen, caffeine, methylphenidate and desmopressin). In case of severe postprandial hypotension, acarbose and octreotide are recommended (strong recommendation, moderate level of evidence). Alternatively,
\end{abstract}

Alessandra Fanciulli

alessandra.fanciulli@i-med.ac.at

1 Department of Neurology, Medical University of Innsbruck, Anichstrasse 35, 6020 Innsbruck, Austria voglibose or caffeine, for which a weak recommendation is available, may be useful.

Keywords Neurogenic orthostatic hypotension . Postprandial hypotension - Syncope - Evidence-based treatment · Grade

\section{Introduction}

Neurogenic orthostatic hypotension $(\mathrm{nOH})$ is a key feature of cardiovascular autonomic failure, defined by a sustained blood pressure (BP) fall $\geq 20 \mathrm{mmHg}$ systolic and/ or $\geq 10 \mathrm{mmHg}$ diastolic within the $3 \mathrm{~min}$ of active standing or head-up tilt.

Prevalence of $\mathrm{nOH}$ is $30 \%$ above 65 years of age (Tilvis et al. 1996) and rises up to $70 \%$ in institutionalized patients (Freeman et al. 2011). nOH occurs in up to $80 \%$ of patients with multiple system atrophy (MSA) (Ha et al. 2011; Köllensperger et al. 2010; Fanciulli and Wenning 2015), 30\% of patients with Parkinson's disease (PD) (Velseboer et al. 2011) and $100 \%$ of patients with pure autonomic failure (PAF) (Goldstein et al. 2015). nOH may also develop in onethird of patients suffering from diabetes mellitus, amyloidosis or spinal cord injury (Low et al. 2004; Wang et al. 2008; Cariga et al. 2002; Illman et al. 2000).

In patients with cardiovascular autonomic failure, a profound and rapid BP fall occurs following food ingestion, due to inability of the sympathetic nervous system to counteract postprandial splanchnic hyperemia, and frequently leads to exacerbation of orthostatic hypotension (Onrot et al. 1985; Mathias et al. 1989, 1991). Post-prandial hypotension, that is a systolic BP drop $>20 \mathrm{mmHg}$ within $2 \mathrm{~h}$ from food ingestion, may accompany $\mathrm{nOH}$ in up 
to $50 \%$ of patients (Frongillo et al. 1995; Senard et al. 1992).

Symptoms of nOH, including recurrent syncope, dizziness, weakness, nausea, tremulousness, headache or "coathanger pain" (pain in the neck and shoulder region) upon standing, may be exacerbated in the early morning, by exercise, heat exposure, dehydration and alcohol consumption. Symptomatic nOH can be severely disabling and poses at higher risk for injurious falls (Rascol et al. 2015). Development of cardiovascular autonomic failure has been also associated with shorter survival, cognitive impairment, as well as higher incidence of cardio- and cerebrovascular events on the long term (Fanciulli et al. 2013).

Management of cardiovascular autonomic failure may result challenging in clinical practice, relying on a combination of non-pharmacological and pharmacological measures, in which the underlying etiology, severity of symptoms and the individual risk-benefit ratio need to be taken into account.

\section{Aim and methods}

In the present paper, we provide an evidence-based overview of non-pharmacological and pharmacological treatment options for $\mathrm{nOH}$ and related symptoms of cardiovascular autonomic failure, i.e., post-prandial hypotension and exercise-induced hypotension.

A systematic keyword-based literature search was performed. Articles published in PubMed between January 1985 and March 2017 were screened. Keyword terms comprised: 'neurogenic orthostatic hypotension', 'postural hypotension', 'postprandial hypotension', 'exercise-induced hypotension' and 'syncope'. These terms were searched alone and in combination with 'non-pharmacological treatment', 'pharmacological treatment', 'therapy' and 'treatment'.

Following selection criteria were adopted:

- English language.

- Study cohorts: Patients with idiopathic nOH (e.g., pure autonomic failure, multiple system atrophy, Parkinson's disease) and, with the exception of spinal cord injury, $\mathrm{nOH}$ secondary to other diseases [e.g., diabetes mellitus, amyloidosis - for classification of primary and secondary nOH, see also Fig. 1 Primary and secondary causes of $\mathrm{nOH}$ according to the site of lesion (Low 2008)].

- Allowed study design: randomized controlled trials, open-label trials, case series and case reports.

Additional data were collected by hand-searching references of selected articles according to the abovementioned criteria.

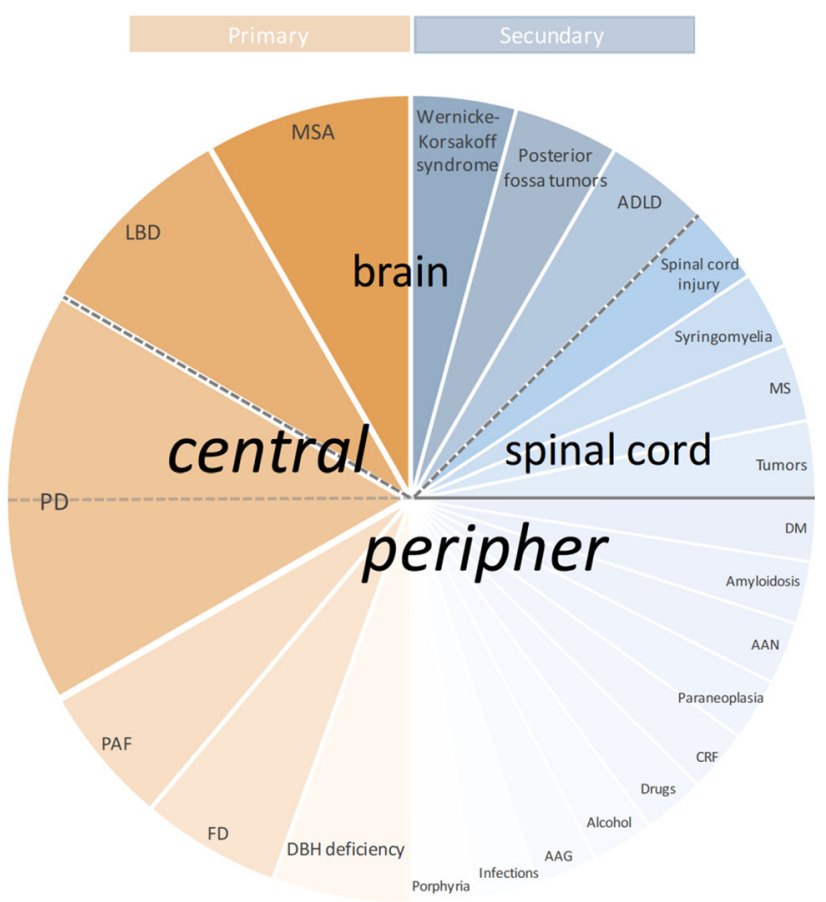

Fig. 1 Primary and secondary causes of $\mathrm{nOH}$ according to the site of lesion-schematic representation. $A A G$ autoimmune autonomic ganglionopathy, $A A N$ autoimmune autonomic neuropathy, $A D L D$ autosomal dominant leukodystrophy, $C R F$ chronic renal failure, $D B H$ deficiency dopamine- $\beta$-hydroxylase deficiency, $D M$ diabetes mellitus, $F D$ familial dysautonomia (=hereditary sensory and autonomic neuropathy type III, Riley-Day syndrome), $L B D$ Lewy-body dementia, $M S$ multiple sclerosis, $M S A$ multiple system atrophy, $n O H$ neurogenic orthostatic hypotension, $P A F$ pure autonomic failure, $P D$ Parkinson's disease

The Grading of Recommendations Assessment, Development and Evaluation (GRADE) approach has been applied for classification of quality of evidence and recommendation level (Leone et al. 2013). The GRADEpro GDT software, provided by the GRADE working group to support grading of quality of evidence and strength of recommendation, has been used for this purpose ("https:// gradepro.org").

\section{Treatment of $\mathrm{nOH}$ and related symptoms: classification of evidence}

\section{Non-pharmacological treatment}

Effects of water drinking on orthostatic, postprandial and exercise-induced hypotension

The short-term effect of rapid water drinking on orthostatic hypotension has been investigated in studies with sample sizes ranging from 5 to 47 patients with autonomic failure. Nine open-label studies, using different study protocols and 
outcomes, that investigated the hemodynamic effect of $300-500 \mathrm{ml}$ water have been identified. Six studies reported a significant pressor effect of water drinking that lasted approximately $35 \mathrm{~min}$ after ingestion of water (Jordan et al. 1999, 2000; Cariga and Mathias 2001; Shannon et al. 2002; Young and Mathias 2004; Deguchi et al. 2007). In contrast, Mathias et al. 1991 reported that $300 \mathrm{ml}$ water drinking did not have an effect on BP when measured 45 min after water ingestion in patients with MSA and PAF (Mathias et al. 1991). Similar findings were also reported in patients with PD and $\mathrm{nOH}$ : ingestion of $500 \mathrm{ml}$ water did not change orthostatic BP when measured $60 \mathrm{~min}$ after fluid intake (Senard et al. 1999).

The effect of rapid water drinking on postprandial hypotension has been evaluated in two studies. In seven patients with $\mathrm{PAF}$, rapid water drinking $(480 \mathrm{ml},<5 \mathrm{~min}$ before test meal) significantly decreased postprandial BP fall (Shannon et al. 2002). A similar effect was also observed in five patients with MSA $(350 \mathrm{ml},<5 \mathrm{~min}$ before test meal) (Deguchi et al. 2007). The effect of water drinking (480 $\mathrm{ml}$ distilled water within $5 \mathrm{~min}$ ) on exerciseinduced $\mathrm{OH}$ has further been evaluated in a small study in 8 patients with PAF. Although water drinking had an effect on post-exercise $\mathrm{OH}$, no benefit has been demonstrated on BP fall during supine exercise (Humm et al. 2008). Although none of the abovementioned studies reported any adverse event, Jordan et al. 1999 commented that water ingestion may cause supine systolic BP increase more than $100 \mathrm{~mm} \mathrm{Hg}$ in some patients with underlying cardiovascular autonomic failure (Jordan et al. 1999).

Quality of evidence The quality of evidence for water drinking to reduce postural BP drop is very low.

Safety issues None.

Recommendation The recommendation for water to stabilize BP in patients with autonomic failure is weak.

\section{Sodium intake}

Few data are available on the effect of salt intake on BP in patients with nOH. Lipp et al. (2005) studied the response to water $(500 \mathrm{ml})$ or to normal saline $(500 \mathrm{ml} \mathrm{NaCl} 0.9 \%)$ administered through nasogastric tube in 10 patients with MSA. Results of this single-blinded crossover study demonstrated that ingestion of water increased systolic BP, whereas normal saline hardly showed any pressor response (Lipp et al. 2005).

Raj et al. (2006) evaluated the benefit of water and salt intake in nine patients with $\mathrm{nOH}$ of unspecified origin in a randomized, crossover trial. Although both water alone $(473 \mathrm{ml})$ and in combination with salt $(2 \mathrm{~g} \mathrm{NaCl})$ increased $\mathrm{BP}$, salt intake attenuated global pressor effect (Raj et al. 2006). One further study investigated the effect of water and salt intake in seven patients with MSA: whereas water intake $(450 \mathrm{ml})$ significantly increased BP values, clear soup intake $(450 \mathrm{ml}$ soup containing $1.7 \mathrm{~g}$ salt) reversed this effect (Z'Graggen et al. 2010).

Quality of evidence Based on available data, the quality of evidence for salt intake to reduce orthostatic BP fall is low. Safety issues None.

Recommendation The recommendation for increased salt intake to stabilize BP in patients with autonomic failure is weak.

\section{Meal size}

The intake of smaller meals may improve postprandial hypotension, since food volume and caloric load play an essential role in determining the degree of postprandial splanchnic hyperemia.

The effect of meal size on postprandial hypotension has been studied in one study by Puvi-Rajasingham and Mathias (1996) in seven patients with autonomic failure due to MSA or PAF. Patients were randomized to start either with three meals per day or six meals per day, providing identical daily caloric intake: larger meals resulted in significant lower BP levels in all positions and aggravation of postprandial orthostatic symptoms compared to small meals. However, the mean orthostatic BP fall was unaffected by meal size (Puvi-Rajasingham and Mathias 1996).

Quality of evidence The quality of evidence of smaller meals to reduce orthostatic BP drop is very low.

Safety issues None.

Recommendation Based on limited data, the recommendation for reduction of meal size is weak.

\section{Sleeping with head-up tilt}

Nocturnal head-up tilt is aimed at reducing nocturnal pressure natriuresis and, therefore, preventing exacerbation of $\mathrm{nOH}$ in the early morning.

Two small-sized, open-label studies have been conducted to investigate the effect of nocturnal head-up tilt $\left(12^{\circ}\right)$ on orthostatic BP in patients with $\mathrm{nOH}$. Ten Harkel et al. (1992) reported that nocturnal head-up tilt alone or in combination with fludrocortisone $(0.1-0.2 \mathrm{mg})$ significantly reduced orthostatic BP fall and improved orthostatic symptoms in six patients with $\mathrm{nOH}$. Study period for each intervention was 1 week, including an average follow-up of 14 months (Ten Harkel et al. 1992). Latter findings were replicated by Van Lieshout et al. (1999) in a 3-week openlabel study in patients with PAF $(n=8)$ : combination of nocturnal head-up sleep and fludrocortisone led to a significant increase in upright BP and minimally improved 
orthostatic tolerance (van Lieshout et al. 1999). Both studies reported that supine BP remained uninfluenced by treatment. Similar results have been reported in one further patient, where chronic volume expansion due to nocturnal head-up sleep and fludrocortisone administration improved symptoms of orthostatic intolerance (van Lieshout et al. 1991).

Quality of evidence Based on limited data, the quality of evidence for nocturnal head-up tilt is very low.

Safety issues None.

Recommendation The recommendation for sleeping with head-up tilt position during night is weak.

\section{Physical countermaneuvers}

Physical countermaneuvers may stabilize orthostatic BP through enhancement of peripheral vasoconstriction during orthostatic stress.

The effect of several countermaneuvers has been investigated in a cohort of 17 patients with familial dysautonomia by Tutaj et al. (2006) in an open-label, randomized crossover trial: bending forward, squatting and abdominal compression $(20 \mathrm{mmHg})$, but not leg crossing, significantly ameliorated orthostatic BP fall in this cohort (Tutaj et al. 2006).

In contrast, leg crossing, but not tiptoeing, was able to combat orthostatic BP fall in patients with $\mathrm{nOH}$ due to PAF (Ten Harkel et al. 1994). Two further open-label studies and one case report are available confirming that a range of physical countermaneuvers attenuate $\mathrm{nOH}$ in chronic autonomic failure (Bouvette et al. 1996; van Lieshout et al. 1992; Wieling et al. 1993). In addition, Smit et al. (2004) evaluated the combination of legs crossing and abdominal compression in an open-label, randomized study. The combination of leg crossing and abdominal compression (20-40 mmHg) was more effective than abdominal compression alone in stabilizing orthostatic BP. Leg crossing alone also improved standing BP in 12 patients with $\mathrm{nOH}$ due to PAF, MSA and diabetes mellitus (Smit et al. 2004).

Quality of evidence The quality of evidence of physical countermaneuvers to reduce orthostatic BP drop in patients with autonomic failure is very low.

Safety issues None.

Recommendation Based on available data, there is a weak recommendation for use of physical countermaneuvers.

\section{Abdominal binders and compression stockings}

The efficacy of compression garments applied to different parts of the body to reduce venous pooling has been assessed in several small-sized studies.
One randomized, single-blind, placebo-controlled, crossover trial in 15 patients with PD and nOH by Fanciulli et al. (2016a) showed that abdominal binders, exerting a compression of $20 \mathrm{mmHg}$, significantly diminished BP fall upon head-up tilt without influencing supine BP values. In a 4-week open-label follow-up, daily use of abdominal binders also significantly improved nOH-related disability, assessed by means of the Orthostatic Hypotension Questionnaire (OHQ) (Fanciulli et al. 2016a).

One further randomized, crossover trial using an openlabel study design has been conducted to evaluate the effect of abdominal compression with conventional and adjustable binders in 13 patients with $\mathrm{nOH}$ by Figueroa et al. (2015): mild abdominal compression $(10 \mathrm{mmHg})$ prior to rising proved to ameliorate $\mathrm{nOH}$. Although an increase in abdominal compression upon standing up to maximal tolerance did not further ameliorate $\mathrm{nOH}$, decompression tended to aggravate orthostatic BP fall (Figueroa et al. 2015). The benefit of abdominal compression has been also reported by Smit et al. (2004) and Tutaj et al. (2006) using an abdominal compression that ranged from 20 to $40 \mathrm{mmHg}$ (see description above).

Studies that systematically evaluated the effect of compression stockings are limited. Denq et al. (1997) showed that either contemporary compression of calves, thighs and low abdomen ( $40 \mathrm{mmHg}$ each) or of the abdomen alone significantly improved $\mathrm{nOH} 14$ patients with MSA, PAF and diabetic neuropathy. In contrast, the sole leg compression (calves and/or thighs) was less effective (Denq et al. 1997). In a young patient with insulin-dependent diabetes mellitus and $\mathrm{nOH}$, symptomatic relief was reported with use of a G-suit (Elizondo et al. 1996).

Quality of evidence The quality of evidence for abdominal binders to reduce orthostatic BP fall is moderate, for compression stockings is very low.

Safety issues None.

Recommendation The recommendation for the use of abdominal binders to improve orthostatic BP fall in patients with autonomic failure is strong, for compression stockings is weak.

\section{Pharmacological treatment}

\section{Orthostatic hypotension}

Midodrine Midodrine, a peripheral $\alpha_{1}$-selective adrenoceptor agonist and prodrug of the active metabolite desglymidodrine, increases blood pressure by inducing vasoconstriction (Pittner et al. 1976).

Three randomized double-blind trials, with either crossover study design (Wright et al. 1998) or parallel group (Jankovic et al. 1993; Low et al. 1997), that directly 
compared the effect of midodrine in a dosage of $2.5-30 \mathrm{mg}$ per day versus placebo, have been conducted. The potent pressor effect of midodrine on standing BP was shown in all three studies with sample sizes ranging from 25 to 171 patients with autonomic failure and a study period (randomized, placebo-controlled phase) lasting up to 4 weeks.

Previously, Kaufmann et al. (1988) had investigated midodrine (25-40 mg/day) in combination with fludrocortisone $(0.1 \mathrm{mg})$ or fludrocortisone alone in a randomized controlled trial with seven patients with MSA and PAF, demonstrating that both combination therapy and fludrocortisone alone were effective in only half of the patients. In the non-responder group, midodrine and fludrocortisone even decreased upright BP with respect to placebo.

Two further randomized, placebo-controlled, comparative studies with a cross-over design are available. FouadTarazi et al. (1995) demonstrated that midodrine administered in a dosage of $2.5-10 \mathrm{mg}$ three times a day, but not ephedrine (6-24 mg/day), significantly improved standing $\mathrm{BP}$ and the ability to stand in eight patients with MSA and PAF.

More recently, midodrine (5-10 mg/day) was compared to atomoxetine $(18 \mathrm{mg} /$ day $)$ in 69 patients with $\mathrm{nOH}$ due to PAF, MSA or PD by Ramirez et al. (2014). Although both treatment arms significantly increased upright BP with respect to placebo, atomoxetine led to a higher increase of standing BP than midodrine. Nonetheless, the lack of a wash-out phase limits interpretation of this study, given that plasmatic half-live of atomoxetine may range from $5 \mathrm{~h}$ (extensive metabolizers) to $21 \mathrm{~h}$ (poor metabolizers) (Sauer et al. 2005).

The effect of midodrine on $\mathrm{nOH}$ has been investigated in further small-sized, non-randomized, open-label studies in patients with autonomic failure of different etiologies [familial dysautonomia (Axelrod et al. 1995), idiopathic $\mathrm{OH}$ (Axenti et al. 1993) and PAF/autonomic neuropathy (Schrage et al. 2004)] supporting efficacy of midodrine for treatment of $\mathrm{nOH}$.

Quality of evidence The quality of evidence of midodrine to reduce orthostatic BP fall in patients with autonomic failure is high.

Safety issues The main safety concern of midodrine is supine hypertension. Further side effects include urinary retention and uncomfortable reactions such as paresthesia, piloerection, chills, scalp and generalized pruritus.

Recommendation Based on available data, there is a strong recommendation for use midodrine for treatment of $\mathrm{nOH}$. Monitoring for exacerbation of supine hypertension and increase of post-void residual urine volume is required.

Droxidopa The efficacy of droxidopa (L-Dihydroxyphenylserine, L-DOPS), a precursor of norepinephrine, has been investigated in five randomized controlled trials involving more than 600 patients with $\mathrm{nOH}$ mainly due to PAF, MSA and PD, in a dosage ranging from 200 to $1800 \mathrm{mg}$, for a placebo-controlled, randomized period of up to 8 weeks.

Whereas four clinical trials demonstrated a significant difference in diverse outcomes favouring droxidopa over placebo [change in supine and upright systolic and diastolic BP (Freeman et al. 1999); supine and standing BP, HR, orthostatic tolerance (Kaufmann et al. 2003); change in OHQ composite score (Kaufmann et al. 2014); change in item 1 (dizziness/lightheadedness) of the Orthostatic Hypotension Symptoms Assessment (OHSA) scale (Hauser et al. 2015)], two further studies failed to meet the primary endpoint, defined as change in OHSA item 1 (dizziness/lightheadedness) (Biaggioni et al. 2015) and change in OHQ composite score (Hauser et al. 2014).

In details, Biaggioni et al. (2015) administered droxidopa, $100-600 \mathrm{mg}$ three times a day, in 101 patients with autonomic failure. The primary efficacy end-point, defined as mean change of OHSA item 1 from randomization to study end, did not significantly differ from placebo (Biaggioni et al. 2015). Nonetheless, a conducted post hoc analysis showed a significant effect of droxidopa measured by OHQ composite score (Biaggioni et al. 2015). The possibility of a substantial carryover effect, explaining the failure of droxidopa in primary outcome, has been discussed, since patients randomized to placebo reported a sustained relief of the orthostatic dizziness/lightheadedness score and standing systolic BP at the end of the study. Besides, symptomatic improvement simply related to study participation has been hypothesized, possibly due to better adherence to non-pharmacological countermeasures during observation period (Kaufmann et al. 2015).

A pre-planned interim efficacy analysis of study $306 \mathrm{~A}$ in 51 patients with PD failed to show a significant difference for droxidopa with respect to placebo in primary endpoint, which was defined as change in OHQ composite score after 8 weeks (Hauser et al. 2014). Primary outcome was, therefore, modified to changes in item 1 of the OHSA score at 1-week-treatment for patients subsequently enrolled in study $306 \mathrm{~B}$. Pooled results from study 306A and B demonstrated significant improvement in the dizziness/lightheadedness OHSA score (item 1) after 1-week-treatment in 171 patients with PD (Hauser et al. 2015).

Several additional small-sized studies (Carvalho et al. 1997; Mathias et al. 2001; Kaufmann et al. 1991; Matsubara et al. 1990; Goldstein and Sharabi 2009) and case reports (Sakoda et al. 1958; Kachi et al. 1988; Man in't Veld et al. 1987; Biaggioni and Robertson 1987) further report benefit from droxidopa in patients with $\mathrm{nOH}$ due to MSA, PAF, PD, familial amyloidotic polyneuropathy and dopamine-beta-hydroxylase deficiency. 
Quality of evidence The quality of evidence of droxidopa to reduce nOH-related symptoms is moderate based on missing long-term data.

Safety issues Possible side effects include hypertension, headache, dizziness and nausea. Falls, urinary tract infections, headache, syncope and dizziness have been reported at open-label follow-up, though systematic post-marketing studies to define rate of adverse events in clinical practice are not available yet (Kaufmann et al. 2015).

Recommendation recommendation for droxidopa to treat orthostatic intolerance in patients with cardiovascular autonomic failure is strong. Regular BP monitoring is required, since treatment may exacerbate supine hypertension.

Fludrocortisone Fludrocortisone, a synthetic mineralocorticoid, increases BP by expanding intravascular volume through sodium and water retention.

Evidence-based data on fludrocortisone for treatment of $\mathrm{nOH}$ are limited. Only two randomized, double-blind, crossover studies are available.

Kaufmann et al. (1988) investigated the effect of fludrocortisone in a dosage of $0.1 \mathrm{mg}$ per day in combination with placebo or midodrine $(25-40 \mathrm{mg} /$ day $)$ in 7 patients with nOH due to MSA or PAF. Fludrocortisone, administered for a period of 1 week, increased upright BP only in half of patients. The remaining patients did not respond to medication and upright BP even decreased (Kaufmann et al. 1988).

Schoffer et al. (2007) compared the efficacy of fludrocortisone and domperidone in a randomized, double-blind comparative study. In 17 patients it was shown that both fludrocortisone $(0.1 \mathrm{mg} /$ day $)$ as well domperidone $(30 \mathrm{mg} /$ d) improved primary outcomes measured by Composite Autonomic Symptom Scale (COMPASS) and clinical global impression of change (CGI) within 3 weeks of treatment. In addition, fludrocortisone and domperidone showed a trend, though not significant, towards reduction of BP fall upon tilt-table testing (Schoffer et al. 2007).

The anti-hypotensive effect of fludrocortisone has been further demonstrated in small-sized, open-label studies in patients with MSA, PD, PAF and hypoadrenergic OH). (Ten Harkel et al. 1992; van Lieshout et al. 1991, 1999; Matsubara et al. 1990; Hakamäki et al. 1998).

Quality of evidence The quality of evidence of fludrocortisone to reduce orthostatic BP fall is very low.

Safety issues Main side effect that has been reported is nocturnal hypertension (Hakamäki et al. 1998). Further adverse events include headache, nausea, dizziness as well as generalized or localized edema and hypokalemia (Ricci et al. 2015). Data for long-term administration are limited. Experimental data suggest that long-term use is associated with end-organ damage (Norcliffe-Kaufmann et al. 2013) and increased risk of renal and cardiac fibrosis (Kaufmann 2016, personal communication).

Recommendation Recommendation for use of fludrocortisone for treatment of $\mathrm{nOH}$ is weak, based on quality of evidence and safety issues. Monitoring of supine BP and electrolytes is required.

Pyridostigmine The therapeutic rationale of the cholinesterase-inhibitor pyridostigmine for treatment of nOH relies on enhancement of cholinergic sympathetic ganglionic transmission, secondarily inducing BP rise. Such effect is especially to be expected during sympathetic activation, i.e., when subjects are in the upright position (Biaggioni 2014).

Based on two prospective open-label trials (Singer et al. 2003, 2006a), Singer et al. (2006b) conducted a doubleblind, randomized, 4-arms (pyridostigmine $60 \mathrm{mg}$ alone, placebo, pyridostigmine $60 \mathrm{mg}$ in combination with 2.5 or $5 \mathrm{mg}$ midodrine), cross-over study. In 58 patients with nOH (MSA, PAF, diabetic and autoimmune autonomic neuropathy), the authors demonstrated that pyridostigmine significantly improved standing diastolic BP one hour after administration. Importantly, no aggravation of supine hypertension was observed (Singer et al. 2006b). In a follow-up, open-label study pyridostigmine maintained favorable effects, especially in patients with less severe nOH without major side effects (Sandroni et al. 2005).

Shibao et al. (2010) evaluated the effect of pyridostigmine $(60 \mathrm{mg})$ versus yohimbine $(5.4 \mathrm{mg})$ in 31 patients with autonomic failure (MSA, PAF, PD) in a single-blind, randomized, controlled trial. In contrast to Singer et al. (2006b), this study could neither confirm that pyridostigmine increases standing diastolic BP $1 \mathrm{~h}$ after drug administration, nor that it improves pre-syncopal symptoms. The authors commented that the study population was more severely affected than in previous studies, partially explaining the conflicting results (Shibao et al. 2010).

Quality of evidence Very low, due to inconsistency of results.

Safety issues Side effects include gastrointestinal symptoms and urinary urgency (Biaggioni 2014).

Recommendation The recommendation for use of pyridostigmine to improve $\mathrm{nOH}$ is weak.

Yohimbine Yohimbine, an $\alpha 2$-adrenergic antagonist, leads to augmentation of sympathetic activity at CNS level, thus increasing norepinephrine release from adrenergic nerve terminals (Biaggioni et al. 1994a).

For yohimbine, three small-sized randomized controlled trials in up to 31 patients with $\mathrm{nOH}$ are available, showing inconsistent results.

Shibao et al. (2010) investigated the effect of yohimbine and pyridostigmine on standing diastolic BP one hour after 
administration in 31 patients with nOH due to MSA, PAF or PD. Only yohimbine $(5.4 \mathrm{mg})$ significantly increased standing diastolic BP, seated systolic and diastolic BP and significantly improved nOH-related symptoms (Shibao et al. 2010). The positive pressor effect of yohimbine has been further reported in small-sized studies $(n=8-35)$ (Jordan et al. 1998, 2000; Onrot et al. 1987) in patients with autonomic failure.

In contrast to abovementioned findings, Senard et al. (1993) did not observe any BP increase at 24-h ambulatory BP monitoring in 17 patients with PD after 4-week treatment with yohimbine (2 $\mathrm{mg}$ three times a day) (Senard et al. 1993).

Similarly, Okamoto et al. (2012) reported negative results in a single-blind, crossover study with 17 patients with $\mathrm{nOH}$ due to PAF and PD: neither yohimbine $(5.4 \mathrm{mg})$, nor atomoxetine $(18 \mathrm{mg})$ alone significantly increased seated systolic BP $1 \mathrm{~h}$ after drug administration or improved orthostatic symptoms. Only co-administration of both yohimbine and atomoxetine increased seated systolic BP and orthostatic tolerance (Okamoto et al. 2012).

Limits of abovementioned RCTs include lack of a washout phase and/or measurement of supine BP.

Quality of evidence The quality of evidence for yohimbine to increase BP and orthostatic tolerance is very low.

Safety issues No side effects reported, effect on supine BP unknown.

Recommendation The recommendation for use of yohimbine is weak, due to very low and inconsistent quality of evidence.

Atomoxetine Atomoxetine, which augments norepinephrine concentration in the synaptic gap by selectively blocking norepinephrine transporter, has been evaluated in three randomized, placebo-controlled trials, with a sample size ranging from 21 to 69 subjects.

As mentioned above, Okamoto et al. 2012 showed that neither atomoxetine $(18 \mathrm{mg})$ nor yohimbine $(5.4 \mathrm{mg}) \mathrm{sig}$ nificantly changed seated systolic BP or orthostatic tolerance $1 \mathrm{~h}$ post-treatment in a cohort of 17 patients with nOH due to PAF and PD. Only the combination of both increased seated systolic $\mathrm{BP}$ and improved $\mathrm{nOH}$-related symptoms (Okamoto et al. 2012).

In contrast, Shibao et al. (2007a) demonstrated that atomoxetine $(18 \mathrm{mg})$ significantly increases seated and standing systolic BP in patients with central autonomic failure (MSA), but not in patients with peripheral autonomic failure (PAF/PD) (Shibao et al. 2007a).

Ramirez et al. (2014) further reported that both atomoxetine (18 mg, single dose) and midodrine (5-10 mg, single dose) significantly increased seated and upright BP with respect to placebo in a randomized, single-blind, crossover trial with 65 patients with nOH due to MSA, PAF, PD or of undetermined origin. Atomoxetine showed a higher increase in standing systolic BP with respect to midodrine. Atomoxetine, but not midodrine, also improved nOH-related symptoms with respect to placebo (Ramirez et al. 2014).

A possible carry-over effect cannot, however, be excluded in above mentioned studies, since washout-phases were not described in detail. The effect of atomoxetine on supine BP was not mentioned.

Quality of evidence Based on available literature, the quality of evidence for atomoxetine to raise standing BP is low.

Safety issues No side effects reported, effect on supine BP remains unclear.

Recommendation The recommendation for atomoxetine for treatment of $\mathrm{nOH}$ is weak, based on quality of evidence and pending safety issues.

Fluoxetine The rationale for use of fluoxetine, a selective serotonin reuptake inhibitor, to improve $\mathrm{nOH}$ is unknown. One small, open-label study in five patients with $\mathrm{nOH}$ of varying etiologies (PAF, renal failure, diabetes mellitus) revealed a positive impact of fluoxetine (20 $\mathrm{mg}$ once daily) on nOH after 6-8 weeks of treatment (Grubb et al. 1994). In addition, in an open-label study, Montastruc et al. (1998) demonstrated that chronic administration of fluoxetine (20 mg; 1-month treatment) significantly reduced orthostatic systolic BP fall and provided symptomatic relief in 14 patients with PD (Montastruc et al. 1998).

Quality of evidence The quality of evidence for fluoxetine to improve nOH is very low.

Safety issues Common side effects are gastrointestinal complaints, headache and dizziness (Wernicke 2004).

Recommendation Based on the quality of evidence, the recommendation for fluoxetine for treatment of $\mathrm{nOH}$ is weak.

Ergot alkaloids Ergotamine, an ergot alkaloid, and its derivate dihydroergotamine increase BP through $\alpha$-adrenergic vasoconstriction of arteries and veins (Perrin 1985).

Ergotamine has been investigated in a small-sized $(n=8)$, placebo-controlled, crossover study. Biaggioni et al. 1990 reported that inhalation of ergotamine (1 puff, $0.36 \mathrm{mg}$ ) proved effective compared to placebo in increasing seated and upright BP, as well as prolonging standing time before onset of nOH-related symptoms (Biaggioni et al. 1990). It is, however, unclear whether patients were randomly assigned to begin either with treatment or placebo and if the study included a wash-out phase. In one additional case report (Siminoski et al. 1988) aerosol administration of ergotamine has been described in a patient with multi-domain autonomic failure. 
Dihydroergotamine, in a dosage ranging from 6.5 to 13 $\mu \mathrm{g} / \mathrm{kg}$, was investigated in two small-sized, randomized, placebo-controlled studies with a crossover design (Hoeldtke et al. 1989b; Hoeldtke and Israel 1989), in which an increase of upright BP was shown. In both studies, main limitation was represented by the lack or rather unclear description of a wash-out phase.

In addition, one open-label study (Victor and Talman 2002) compared intravenous administration of ergotamine $(0.15 \mathrm{mg})$ versus oral clonidine $(0.4 \mathrm{mg})$ in four patients with severe autonomic failure: whereas both drugs increased supine BP, dihydroergotamine was more effective in contrasting orthostatic BP fall.

Quality of evidence: Based on limited data, the quality of evidence for both ergotamine and dihydroergotamine to reduce orthostatic BP fall in patients with autonomic failure is very low.

Safety issues: common side effects of ergot alkaloids are nausea, vomiting, paresthesias and fatigue. Severe adverse effects include fibrosis (retroperitoneal, cardiac, pleural, pulmonary), peripheral vasoconstriction and ergotism (Schiff 2006).

Recommendation: in consideration of quality of evidence and safety issues, the recommendation for ergotamine and dihydroergotamine for treatment of $\mathrm{nOH}$ is weak.

Recombinant erythropoietin Erythropoietin is reckoned to combat $\mathrm{nOH}$ by stimulating red cell mass production, thus increasing circulating blood volume and tissue oxygenation.

No randomized controlled trials are available for the treatment of $\mathrm{nOH}$ with erythropoietin in the setting of autonomic failure. Several small-sized (sample size up to 24 patients), open-label studies and case reports in patients with primary autonomic failure or $\mathrm{nOH}$ secondary to diabetes mellitus type 1 or familial amyloidosis reported a beneficial effect of erythropoietin on $\mathrm{nOH}$ (Hoeldtke and Streeten 1993; Perera et al. 1995; Biaggioni et al. 1994b; Winkler et al. 2001; Beirão et al. 2008; Kawakami et al. 2003).

Quality of evidence The quality of evidence for erythropoietin to increase orthostatic BP in patients with autonomic failure is very low.

Safety issues Adverse events include flu-like symptoms, allergic reactions, hypertension and increased risk of thrombosis, among others (Eagleton and Littlewood 2003).

Recommendation The recommendation for erythropoietin to treat $\mathrm{nOH}$ in patients with autonomic failure is weak.

Ephedrine Available evidence for ephedrine, a nonspecific direct and indirect $\alpha$ - and $\beta$-adrenoceptor agonist (Fouad-Tarazi et al. 1995), is scarce.
Efficacy and safety of ephedrine have been compared to midodrine in a randomized, double-blind, placebo-controlled, crossover study in eight patients with PAF and MSA by Fouad-Tarazi et al. (1995): ephedrine neither improved standing BP, nor the ability to stand. In contrast, ephedrine significantly increased supine systolic and diastolic BP (Fouad-Tarazi et al. 1995).

Quality of evidence Based on limited data, the quality of evidence for ephedrine to improve standing BP is very low.

Safety issues Adverse events include exacerbation of supine hypertension, dizziness, lightheadedness, photosensitivity and loss of balance.

Recommendation In consideration of quality of evidence, efficacy and safety profile, the recommendation for ephedrine to treat orthostatic hypotension in patients with autonomic failure is weak.

Other ephedra alkaloids Phenylpropanolamine was investigated in an open study in 14 patients with nOH of varying etiologies (PAF, MSA, diabetes mellitus). Administration of 12.5 to $25 \mathrm{mg}$ phenylpropanolamine led to a significant increase in seated $\mathrm{BP}$ in patients with autonomic failure (Biaggioni et al. 1987).

Jordan et al. (1998) used a single-blinded, placebocontrolled design to administer various pressor agents, including phenylpropanolamine. Phenylpropanolamine in a dosage of 12.5 to $25 \mathrm{mg}$ elicited a significant pressor effect with respect to placebo. Notwithstanding, this study was not primarily designed for therapeutic purposes and presents several limitations: randomization is not mentioned; due to individual contraindications, not every patient received each study medication and no clear description of wash-out phases is provided. The authors further state that the study was carried over a period of several years (Jordan et al. 1998).

Jordan et al. (2004) confirmed in an open-label study in 13 patients with autonomic failure that phenylpropanolamine (12.5 and $25 \mathrm{mg}$ ) and pseudoephedrine (a stereoisomer of ephedrine, $30 \mathrm{mg}$ per os) significantly increased BP. The pressor effect was increased by concomitant ingestion of water (Jordan et al. 2004).

Quality of evidence Based on available data, the quality of evidence for phenylpropanolamine and pseudoephedrine to increase BP in the context of cardiovascular autonomic failure is very low.

Safety issues Possible side effects include supine hypertension, anxiety, tremulousness, cerebral and cardiovascular events (Freeman 2008).

Recommendation In consideration of quality of evidence and safety issues, recommendation for phenylpropanolamine and pseudoephedrine for the treatment of $\mathrm{nOH}$ is weak. 
Octreotide Various studies have been identified that investigate the efficacy of octreotide, a somatostatin ana$\log$, for treatment of $\mathrm{nOH}$.

Although Hoeldtke and Israel 1989 observed a beneficial response to octreotide administration $(0.2-26 \mu \mathrm{g} / \mathrm{kg})$ in 28 patients with PAF, MSA and diabetes mellitus, inconsistency among the different study protocols applied, limits interpretation of results (Hoeldtke and Israel 1989).

Bordet et al. (1995) studied the effect of acute administration of octreotide $(100 \mu \mathrm{g})$ in nine patients with MSA in an randomized, placebo-controlled, double-blind study. Octreotide increased duration of head-up tilt test and time until minimal BP values was reached. Notably, octreotide also raised supine BP values (Bordet et al. 1995).

The effect of octreotide on exercise-induced hypotension in the supine position was evaluated by Smith et al. (1995), demonstrating no improvement of it. Nonetheless, octreotide improved orthostatic BP fall pre- and post-exercise (Smith et al. 1995).

Data for administration of octreotide on a regular basis are limited. In an open-label study, Bordet et al. 1994 evaluated the effect of a 6-month octreotide administration in 5 patients with MSA, reporting beneficial effects (Bordet et al. 1994).

Quality of evidence The quality of evidence for octreotide to reduce orthostatic BP fall is low.

Safety issues Side effects include gastrointestinal symptoms, in particular nausea and cramps, facial flushing and hyperglycemia. For this reason, use of octreotide is not recommended in patients with diabetes mellitus, especially in case of concomitant gastroparesis diabeticorum. Supine hypertension has been also reported.

Recommendation Recommendation for octreotide to treat $\mathrm{nOH}$ is weak.

Other agents For a wide range of other compounds, scattered evidence is available. Indomethacin, an inhibitor of prostaglandin synthesis, was studied with different study protocols by Jordan et al. (1998) in 35 patients with nOH of diverse etiologies. Indomethacin $(50 \mathrm{mg})$ elicited a profound pressor effect. In the same study, no significant difference compared to placebo could be demonstrated for ibuprofen, caffeine and methylphenidate (Jordan et al. 1998).

The effect of the vasopressin analog, desmopressin (2-4 $\mu \mathrm{g}$, intramuscular administration), on nocturnal polyuria was measured in a small cohort of five patients with autonomic failure. Desmopressin reduced nocturnal polyuria and prevented early morning orthostatic BP fall, but also increased supine BP (Mathias et al. 1986). Similarly, Sakakibara et al. (2003) showed that intranasal desmopressin reduced nocturia in three patients with MSA; in this study no hypertension was observed (Sakakibara et al. 2003).

Quality of evidence The quality of evidence for the following compounds to reduce nOH is very low: indomethacin, ibuprofen, caffeine, methylphenidate and desmopressin.

Safety issues Based on limited data, safety issues remain unclear.

Recommendation Recommendation for indomethacin, ibuprofen, caffeine, methylphenidate and desmopressin for treatment of $\mathrm{nOH}$ is weak.

\section{Postprandial hypotension}

$\alpha$-Glucosidase inhibitor Acarbose decreases release of vasodilatory gastrointestinal hormones through inhibition of $\alpha$-glucosidase in the small intestine (Shibao et al. 2007b).

The effect of acarbose in treatment of postprandial hypotension has been evaluated in two randomized placebo-controlled trials in patients with PAF or PD (Shibao et al. 2007b) and patients with type 2 diabetes mellitus (Madden et al. 2015).

Acarbose (50-100 mg) was shown to significantly improve postprandial systolic BP fall compared to placebo. Limitation of these studies included a small sample size ( $n=9-15)$ and short-term use (single administration) of acarbose.

Favorable effects were also demonstrated in an openlabel study in patients with MSA $(n=14)$ (Fukushima et al. 2013) and case reports (type 1 diabetes mellitus) (Maule et al. 2004) (type 2 diabetes mellitus) (Yamamoto et al. 2006; Sasaki et al. 2001).

Voglibose, another $\alpha$-glucosidase inhibitor, inhibited postprandial hypotension in a small-sized study $(n=11)$ with pre-post design in MSA, PD, diabetes mellitus and elderly patients (Maruta et al. 2006).

Quality of evidence The quality of evidence for acarbose to reduce postprandial $\mathrm{BP}$ fall is moderate, for voglibose is very low.

Safety issues Most frequent adverse events include gastrointestinal symptoms (e.g. flatulence, diarrhea, stomachache), which have been reported in a meta-analysis, where $\alpha$-glucosidase inhibitors were administered in patients with type 2 diabetes mellitus for glycemic control (Van de Laar et al. 2005). In abovementioned studies, hypoglycemia was not reported either in patients with or without diabetes mellitus

Recommendation In consideration of quality of evidence, efficacy and safety profile, acarbose is strongly recommended to improve postprandial hypotension in patients with autonomic failure. Based on quality of evidence, the recommendation is weak for voglibose. 
Octreotide Octreotide, a somatostatin analog, combats postprandial hypotension by reducing postprandial splanchnic hyperemia induced by gastrointestinal vasodilatory peptides (Raimbach et al. 1989).

Three randomized controlled trials (Hoeldtke et al. 1986a, 1989a, 1998) have been conducted in patients with autonomic failure (mainly due to PAF, MSA or diabetes mellitus) investigating the effect of octreotide in a dosage ranging from 0.1 to $0.6 \mu / \mathrm{kg} /$ day on postprandial hypotension. Although these studies were limited by a small sample size $(n=8-16)$, a favorable effect of the somatostatin analogue was shown in the short-term.

Hoeldtke et al. (1986a, 1989a, b, 1998) demonstrated in all three studies that somatostatin prevented postprandial BP fall. These findings were replicated in further interventional studies with open-label design (Raimbach et al. 1989; Alam et al. 1995; Armstrong and Mathias 1991) and one case report (Hoeldtke et al. 1986b).

Quality of evidence Based on available data, the quality of evidence for octreotide to reduce postprandial BP fall is moderate.

Safety issues Side effects include gastrointestinal symptoms, in particular nausea and cramps, facial flushing and hyperglycemia. For this reason, use of octreotide is not recommended in patients with diabetes mellitus, especially in case of concomitant gastroparesis diabeticorum. Supine hypertension has been also reported.

Recommendation In consideration of moderate quality of evidence, efficacy and safety profile, recommendation is strong for octreotide to treat postprandial hypotension in patients with autonomic failure, with the exception of diabetes mellitus.

Caffeine Caffeine inhibits adenosine-induced splanchnic vasodilatation and should therefore diminish BP fall after food ingestion (Onrot et al. 1985).

The effect of caffeine on postprandial hypotension has been evaluated only in one small-sized randomized controlled trial $(n=5)$ in patients with autonomic failure (diabetes mellitus, PAF, autonomic neuropathy due to alcoholism). Patients were treated with either caffeine $250 \mathrm{mg}$, subcutaneous dihydroergotamine $(10 \mu \mathrm{g} / \mathrm{kg})$, a combination of both or placebo: whereas combination of treatments prevented patients from postprandial hypotension, monotherapy improved postprandial BP fall only partially (Hoeldtke et al. 1989b).

Onrot et al. (1985) further studied the effect of caffeine in a dosage of $250 \mathrm{mg}$ in a small cohort $(n=6)$ of patients with autonomic failure. In this study, which did not include a placebo arm, caffeine showed a favorable single-dose effect on postprandial hypotension, which was maintained after 7-day administration (Onrot et al. 1985).
Quality of evidence Based on available literature, quality of evidence for caffeine to treat postprandial hypotension is very low.

Safety issues No side effects reported.

Recommendation Based on very low quality of evidence, the recommendation for treating post-prandial hypotension with caffeine is weak.

\section{Remarks on classification of evidence}

Tables 1, 2, 3 summarize reviewed studies for non-pharmacological and pharmacological management of $\mathrm{nOH}$ and post-prandial hypotension, while Table 4 provides an overview of quality of evidence, recommendation and safety issues.

Three main aspects penalize the quality of evidence for therapeutic interventions: first, studies are mostly smallsized and include populations of patients with $\mathrm{nOH}$ of different etiologies, which limits information on safety and efficacy in single diseases; second, for some non-pharmacological interventions (e.g., water, meal size, nocturnal head-up tilting, physical countermaneuvers) it is not possible to achieve a placebo-controlled study design; third, replication in larger placebo-controlled randomized clinical trials are often missing for those pharmacological measures, which showed positive results in pilot studies-such lack of data mirrors the challenges of running clinical trials in orphan diseases, where multi-center, adequately powered studies are hindered by limited commercial interest (Kaufmann et al. 2015).

\section{Approach to $\mathrm{nOH}$ and post-prandial hypotension in clinical practice}

Cornerstone of therapy includes both non-pharmacological and pharmacological measures applied in a stepwise manner.

Non-pharmacological treatment options remain pivotal for the treatment of autonomic failure (see Table 5). With exception of abdominal binders (high quality of evidence, strong recommendation) the quality of evidence for all other non-pharmacological interventions is low to very low, and recommendation therefore weak. However, given the favorable safety profile of non-pharmacological interventions, patients should be advised and motivated to apply such strategies on a regular basis. Conservative interventions include adequate fluid (1.5-2 1/day) and salt (6-10 g/day) intake (Biaggioni 2014), as well as abdominal binders, alone or in combination with compression stockings to reduce venous pooling. Bolus ingestion of $500 \mathrm{ml}$ water may diminish BP fall in the following $30 \mathrm{~min}$, and can be therefore purposely applied by the patient based on 


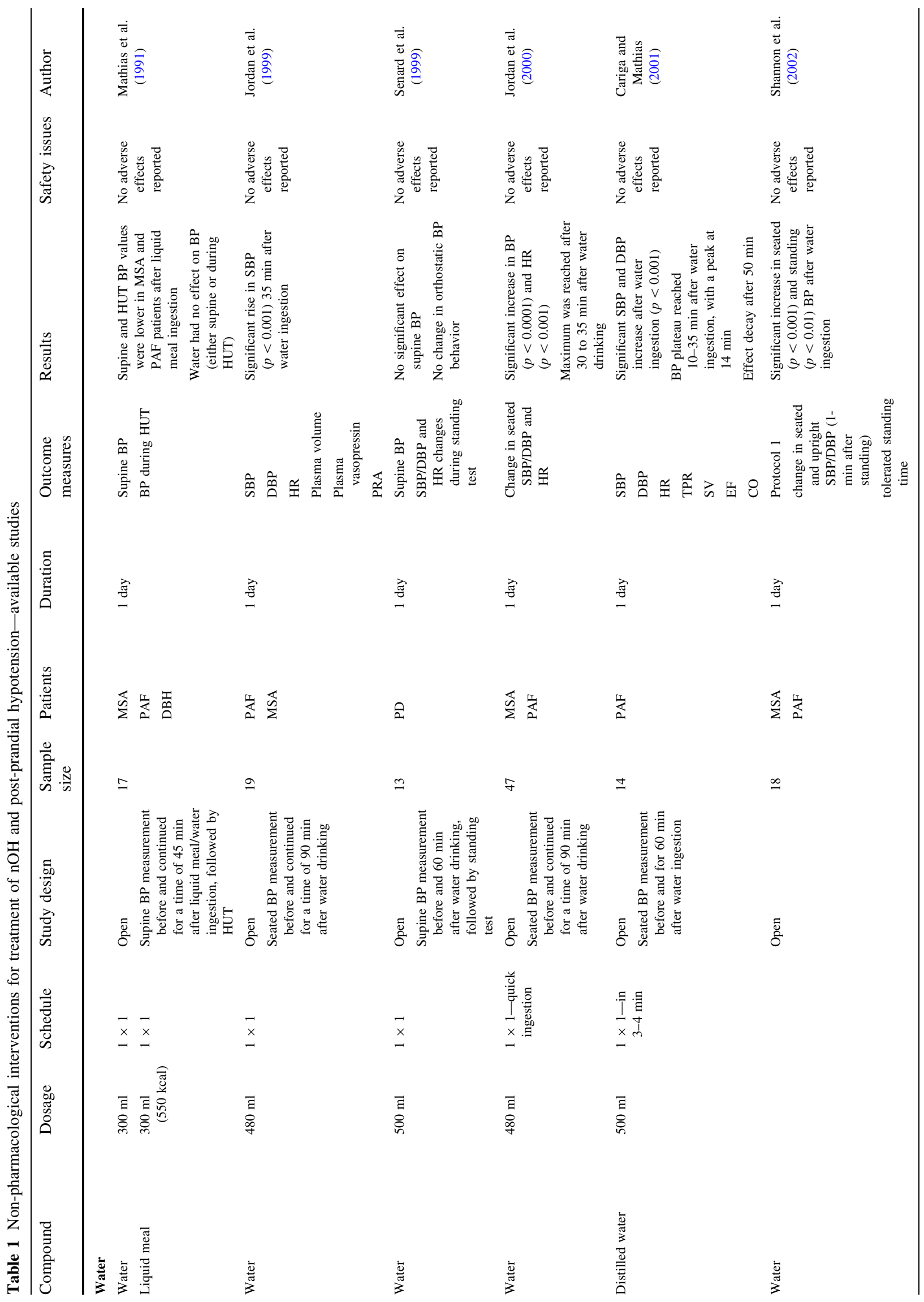




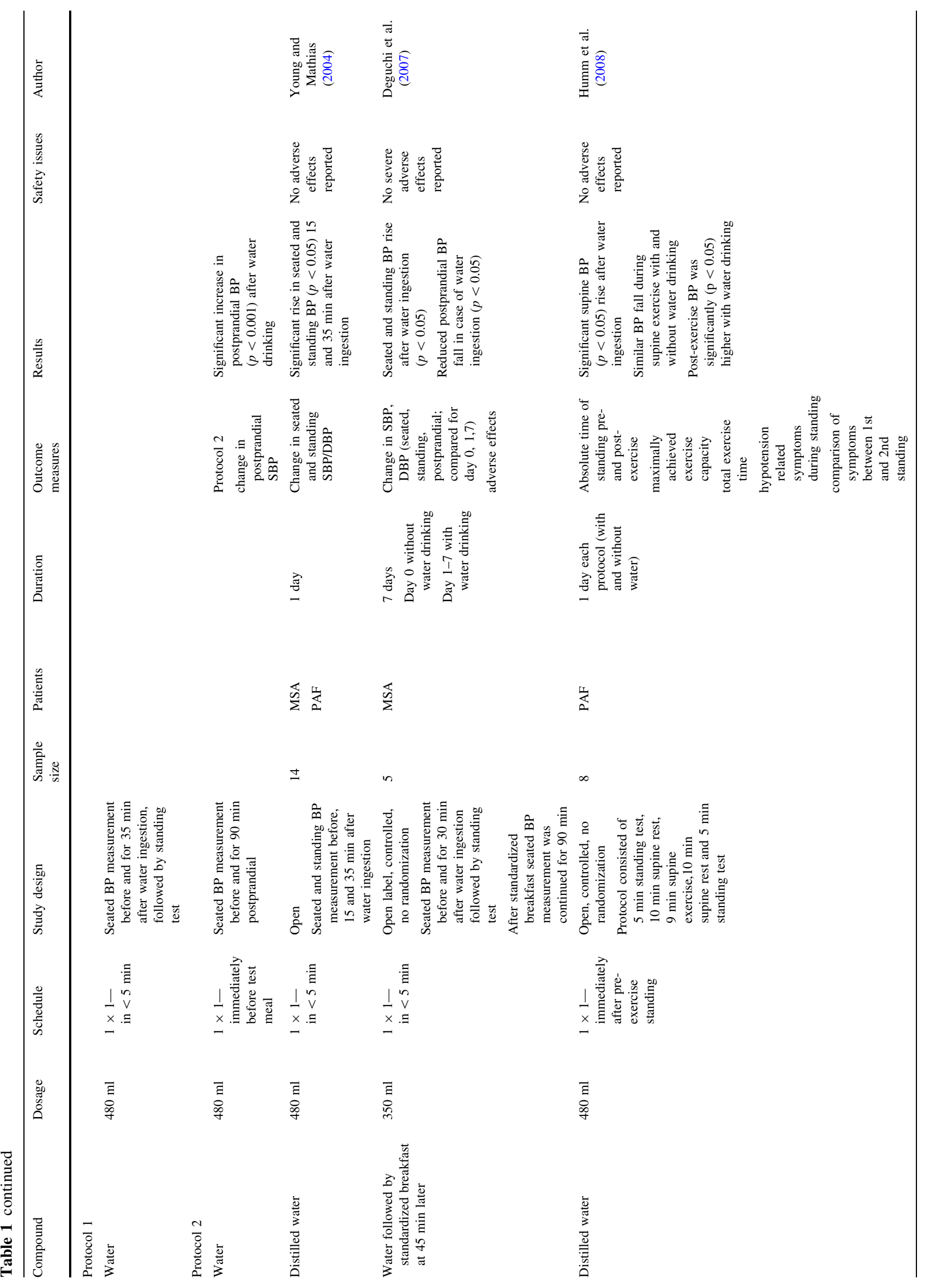




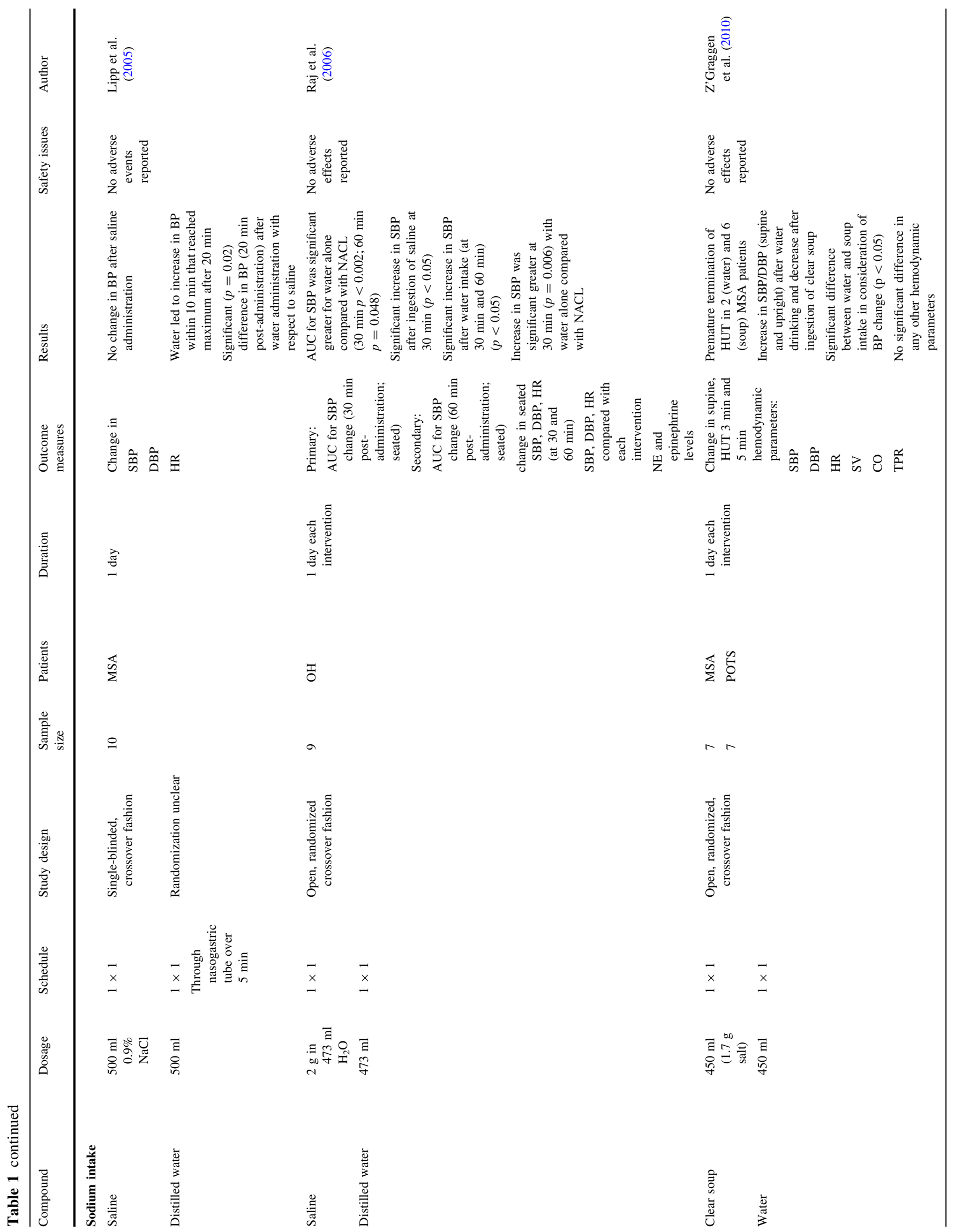




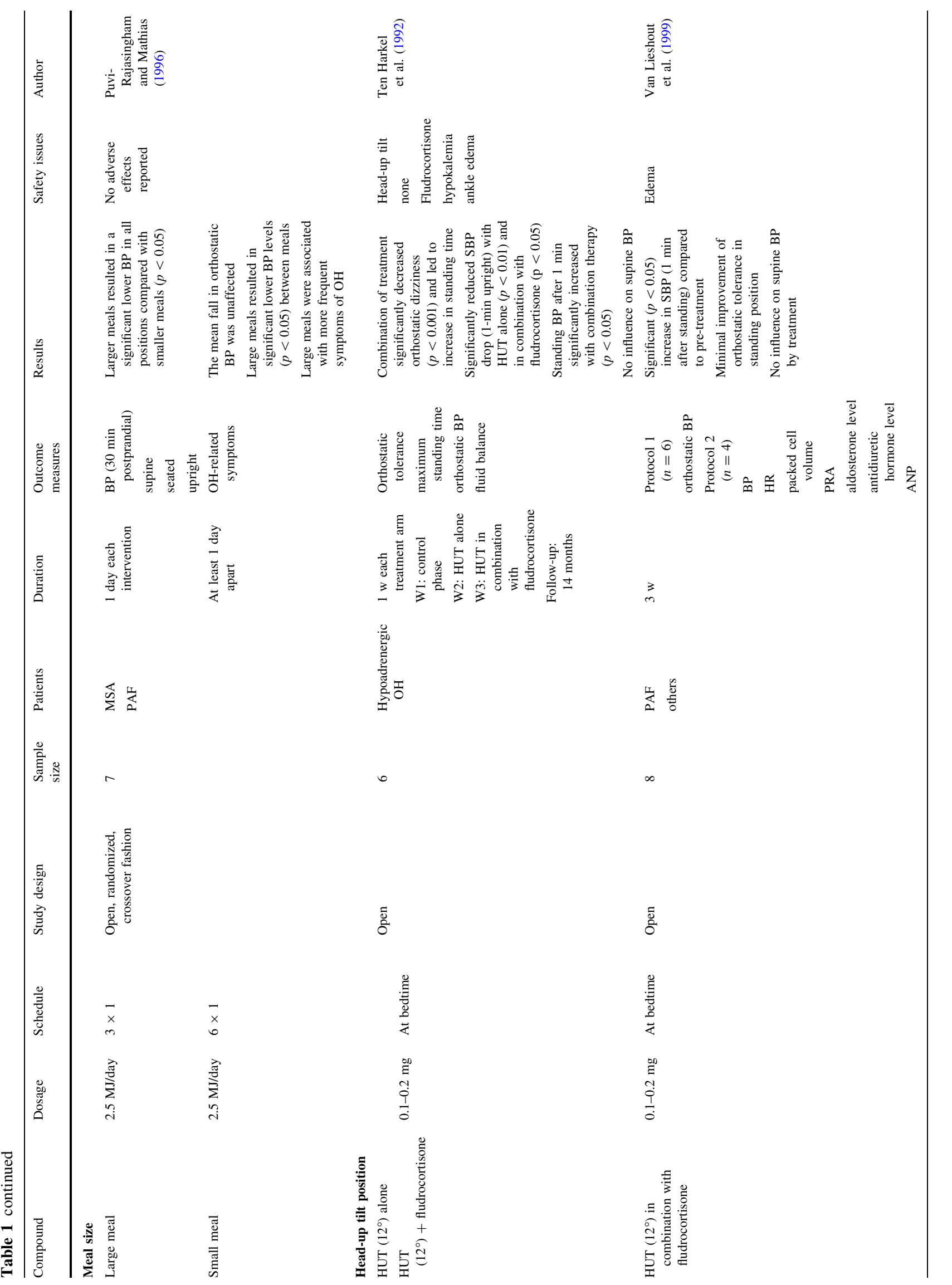




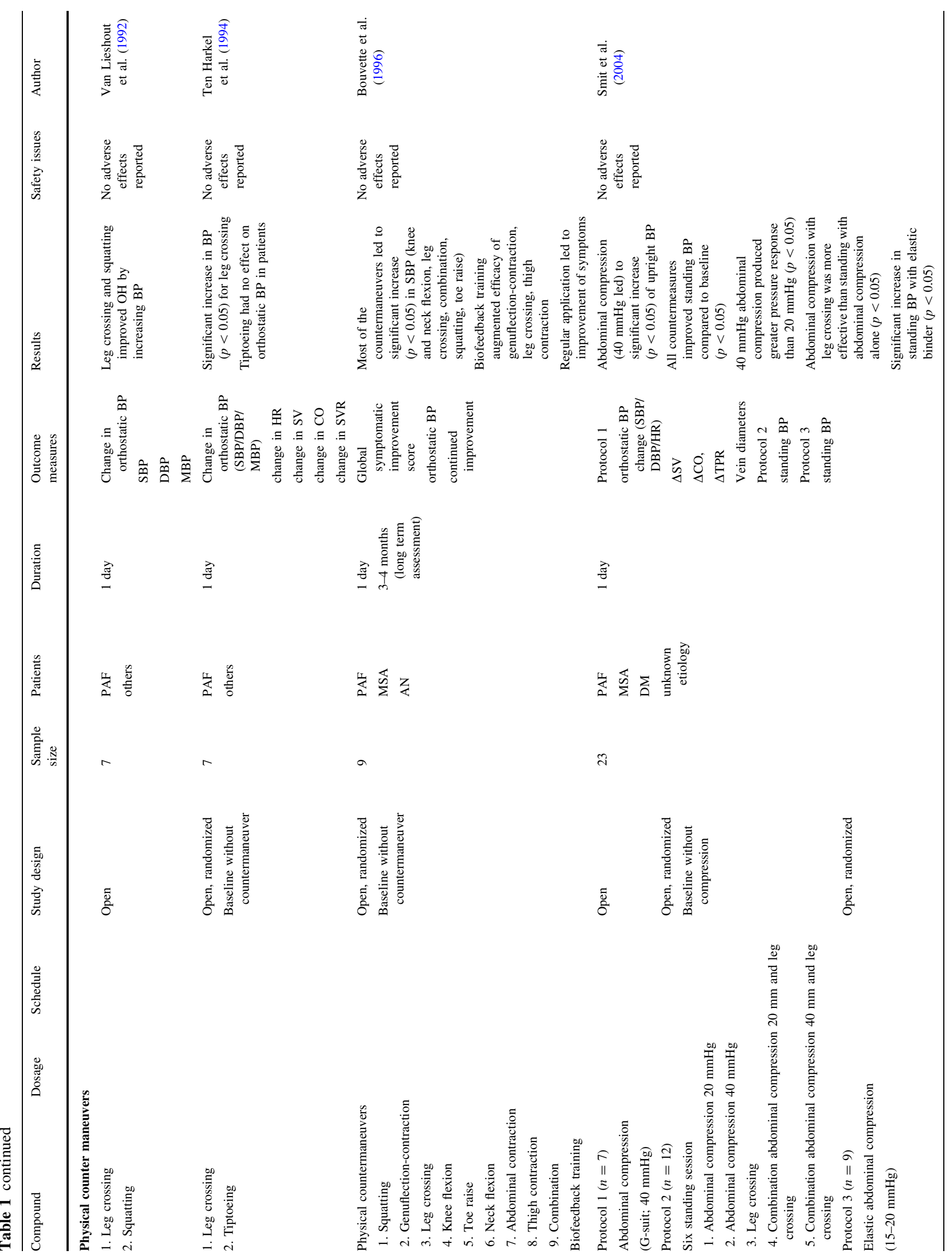




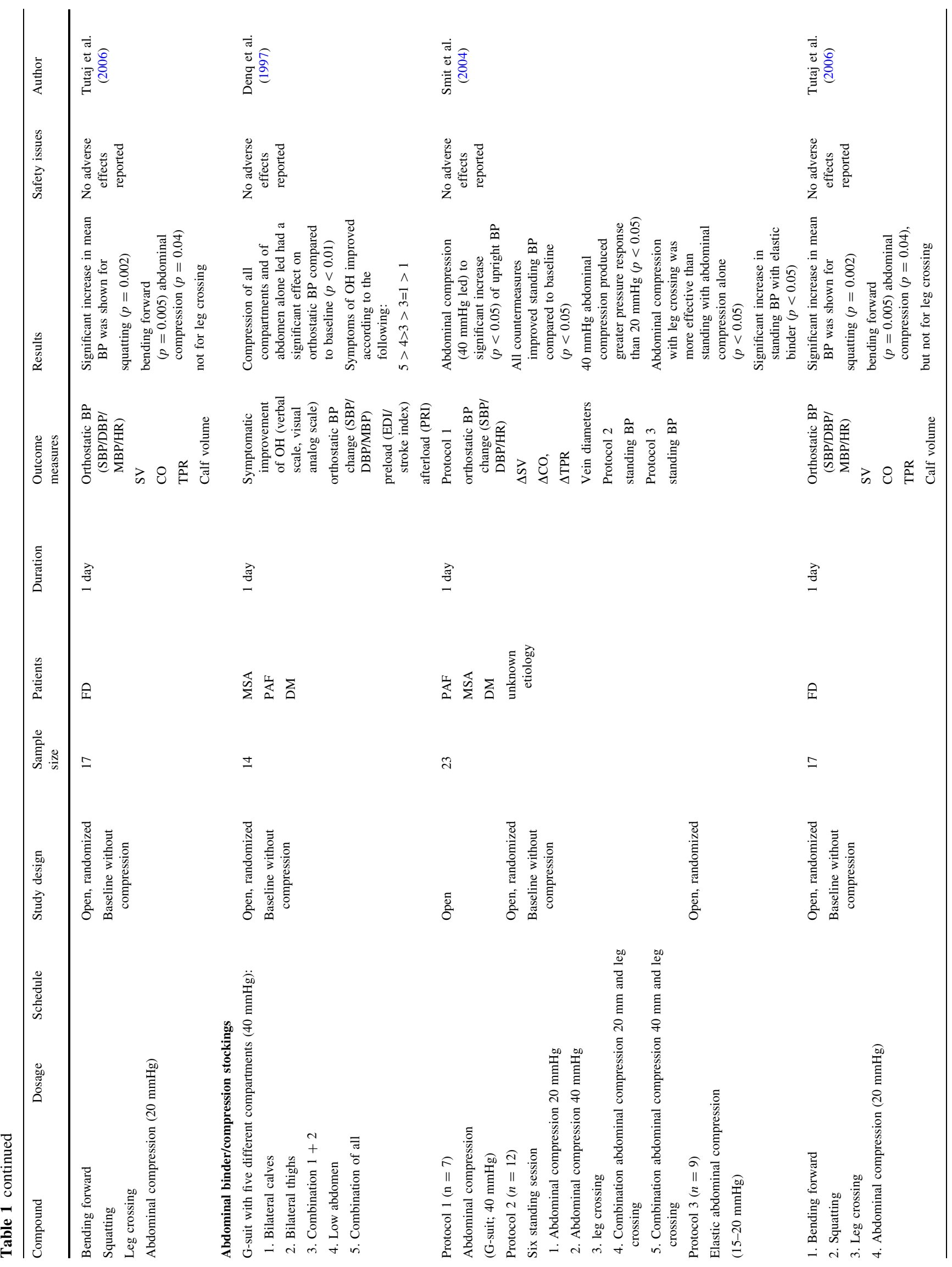




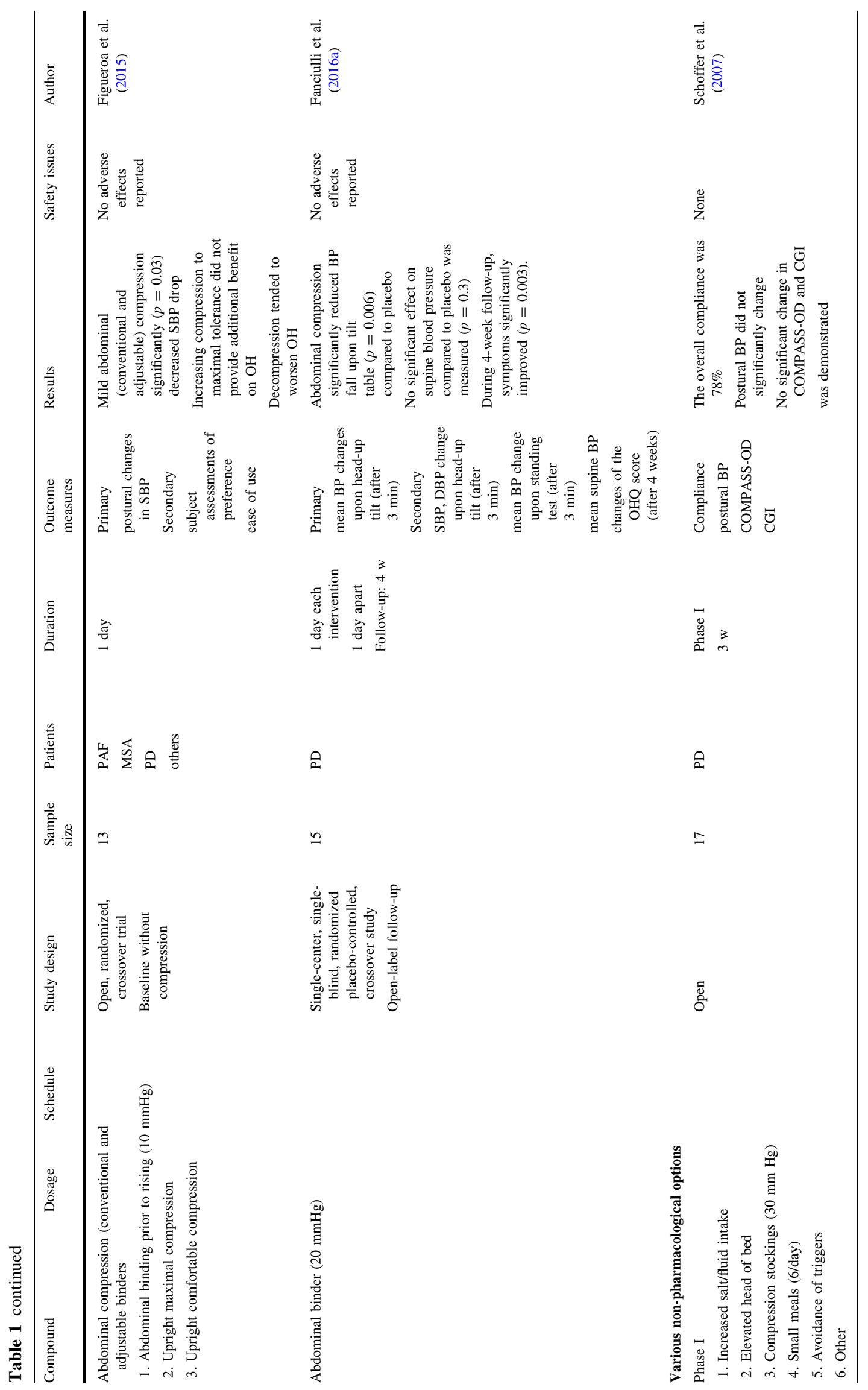




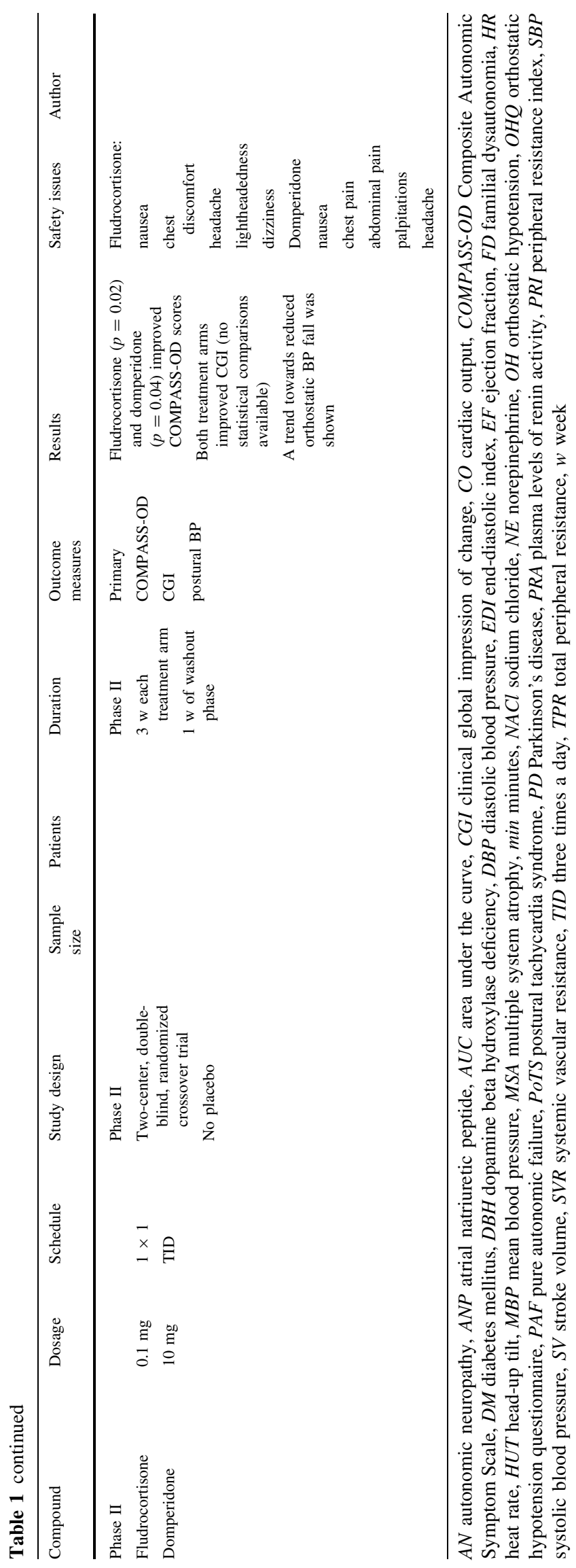




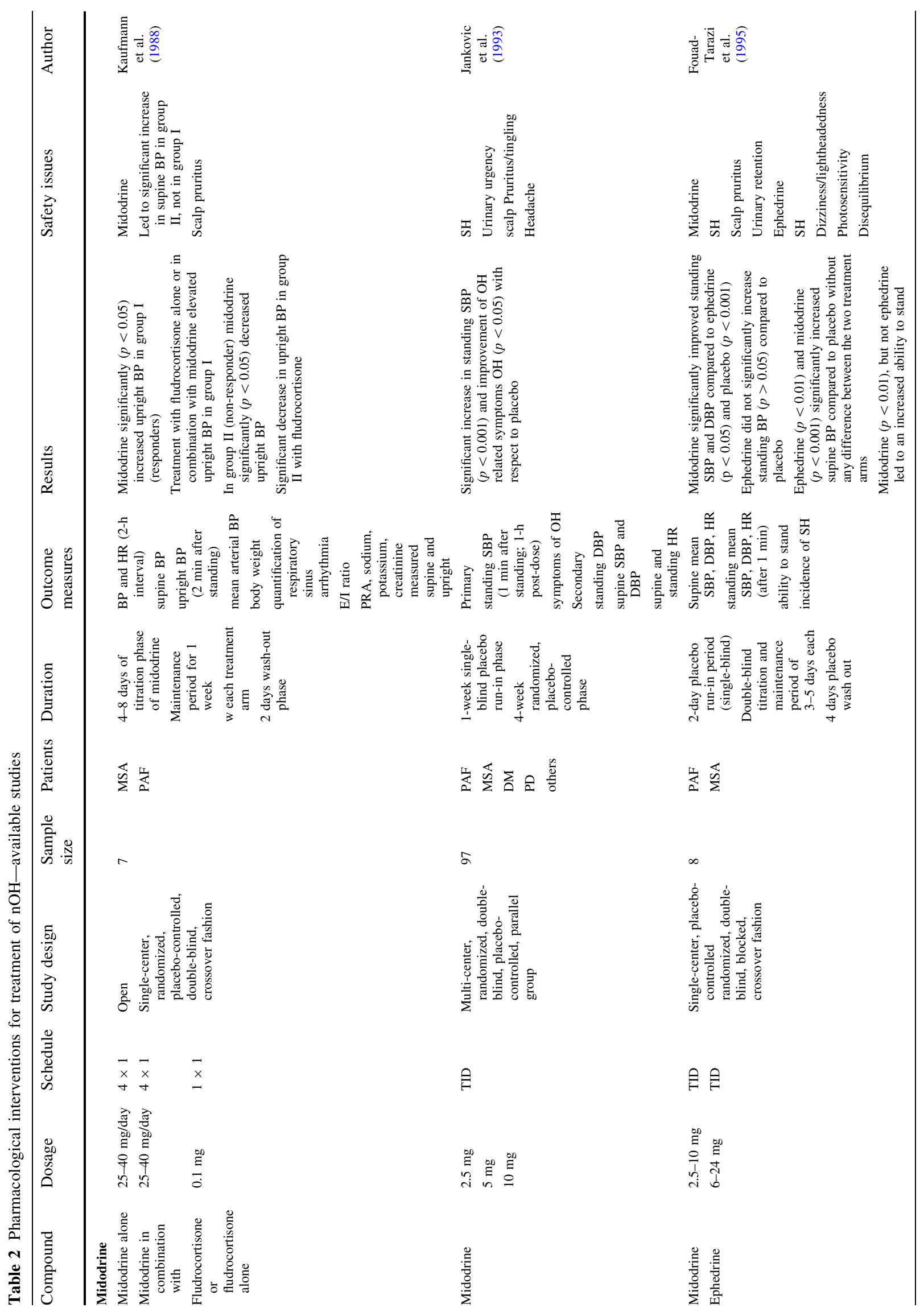




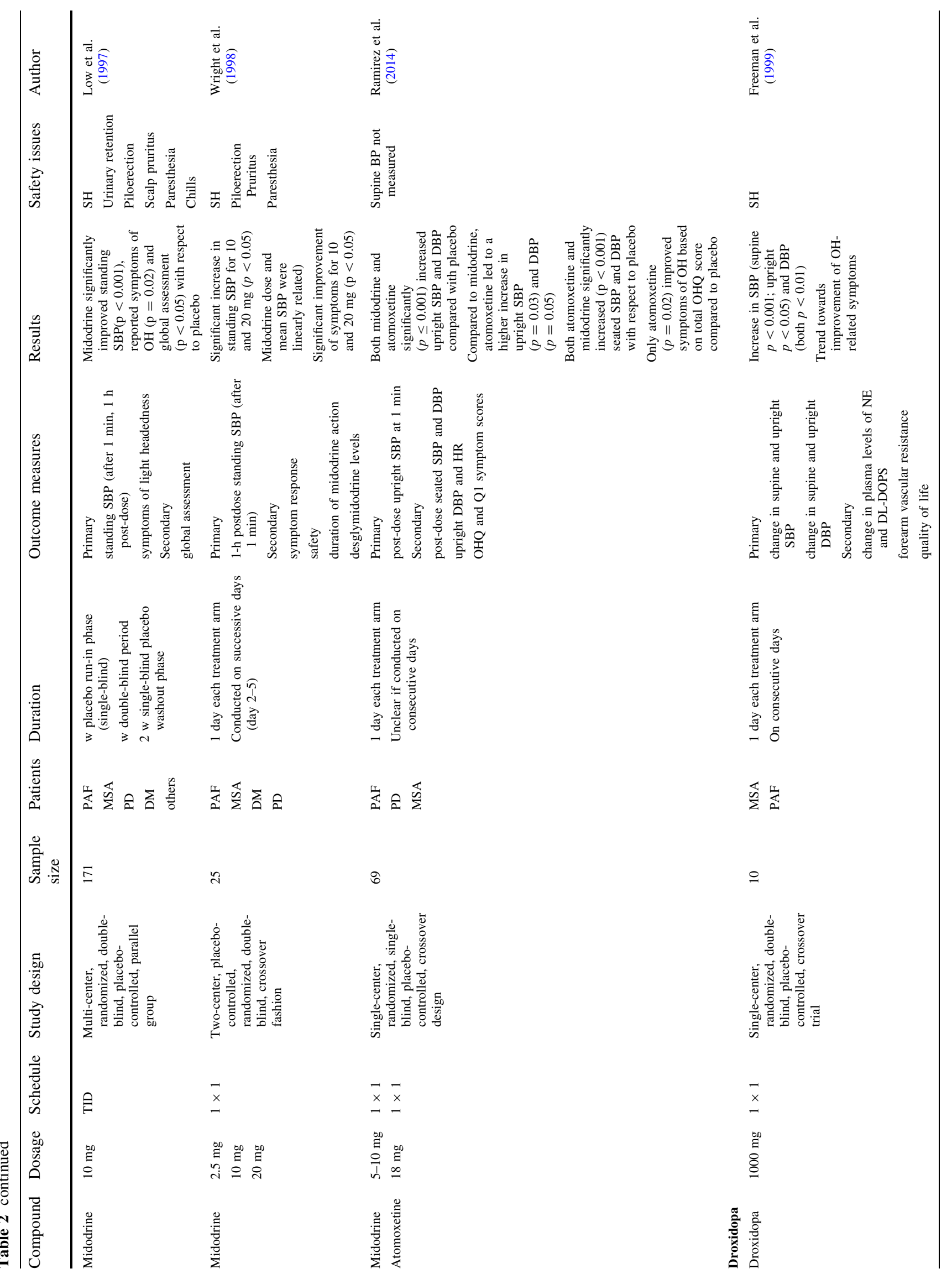




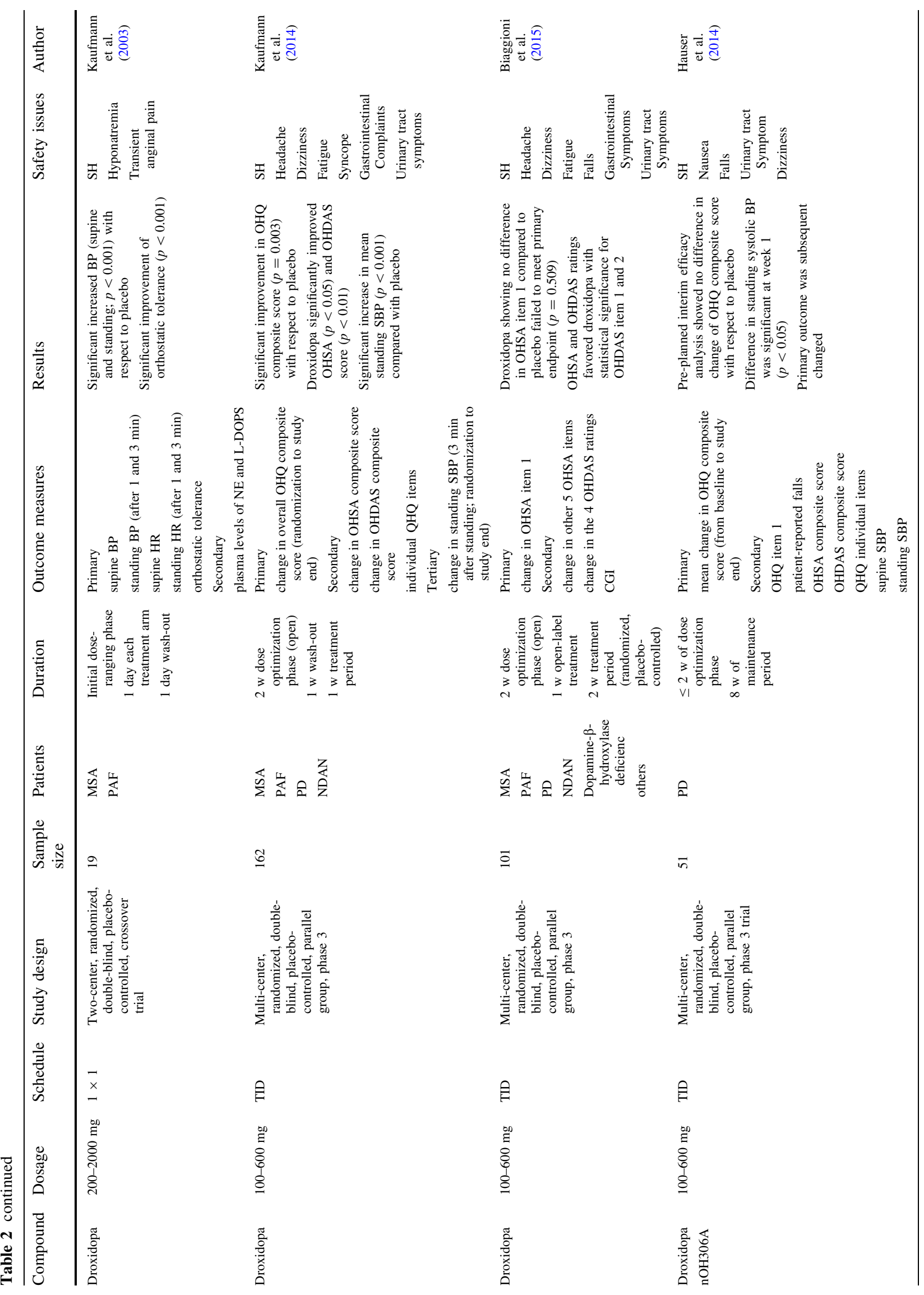




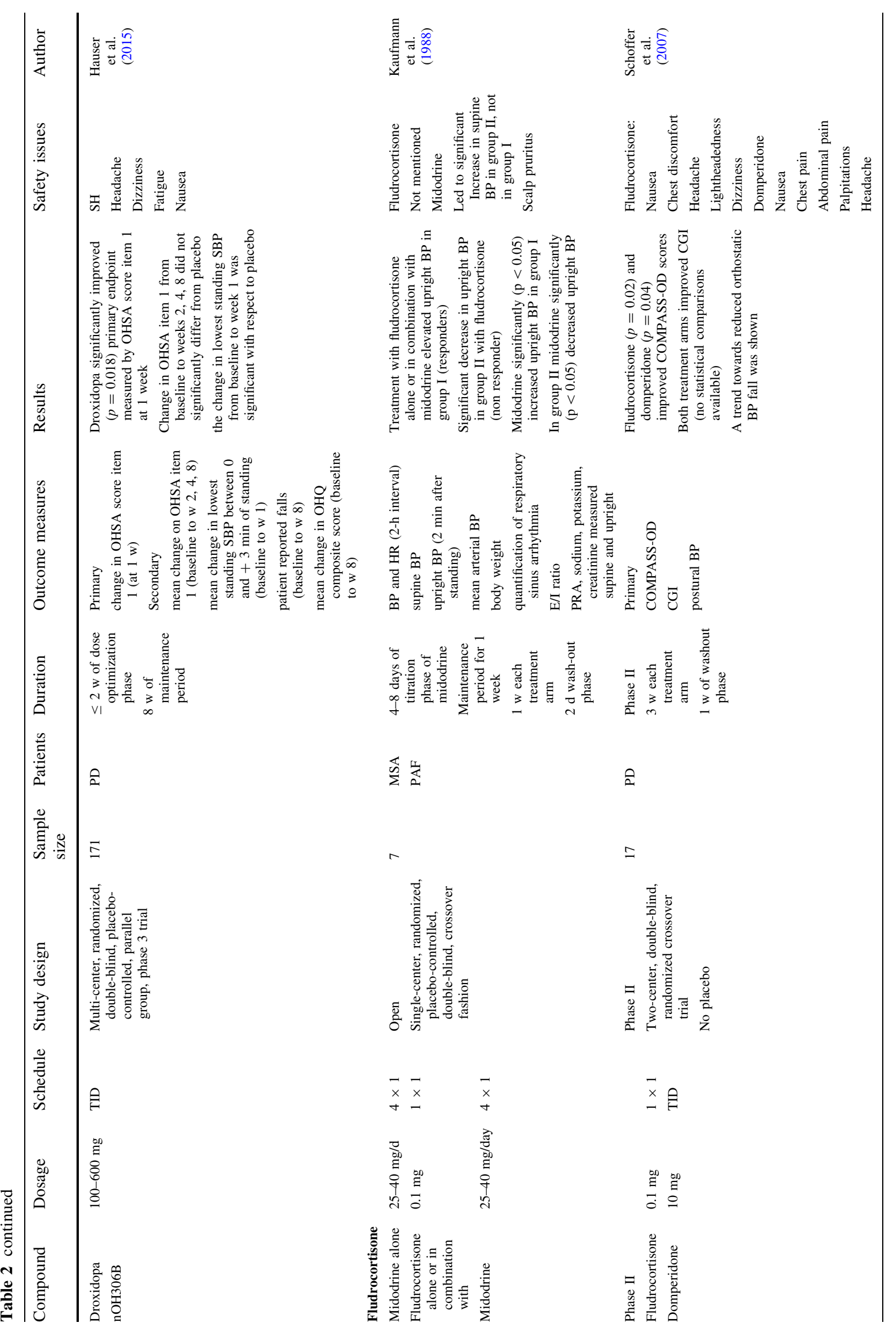




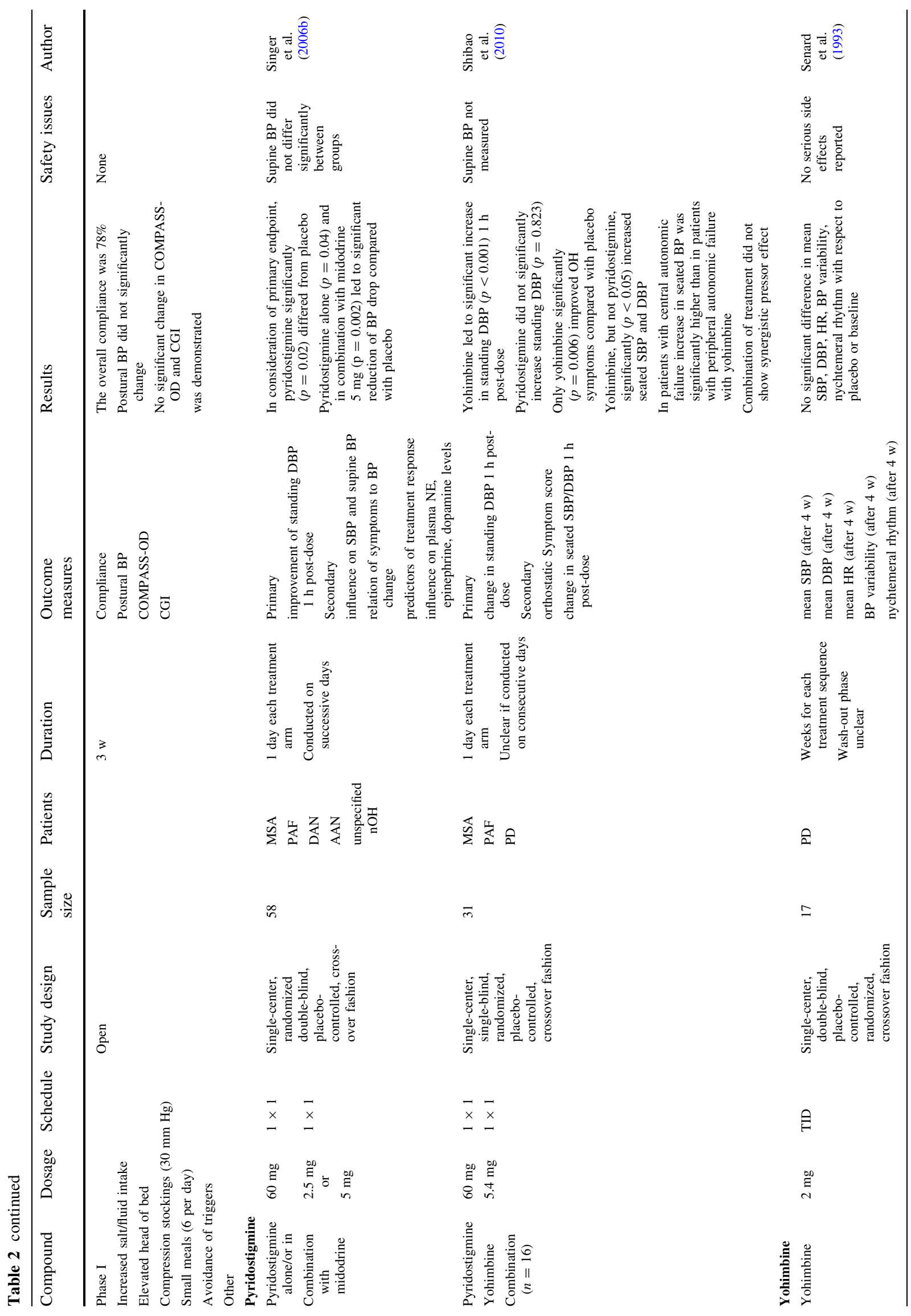




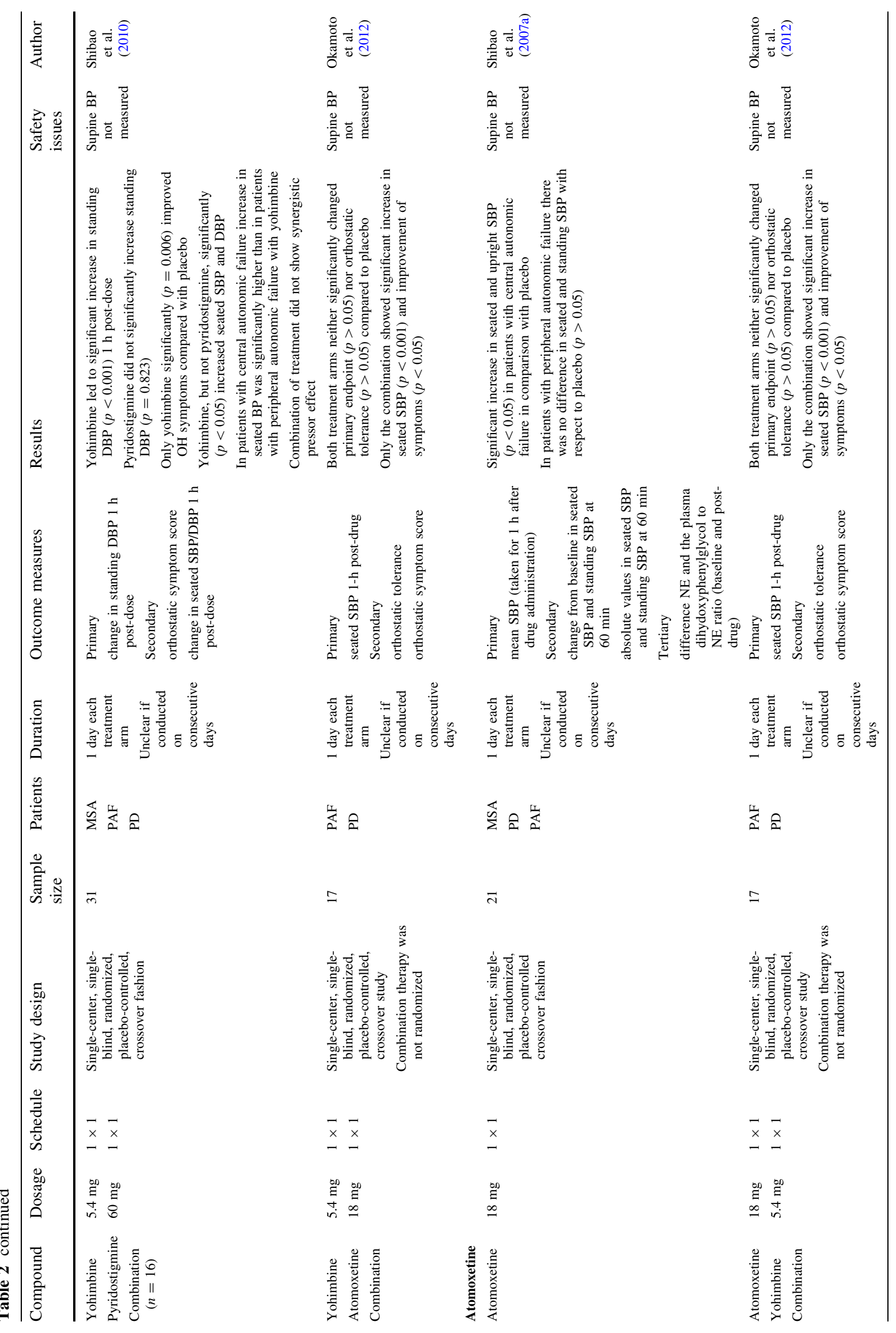




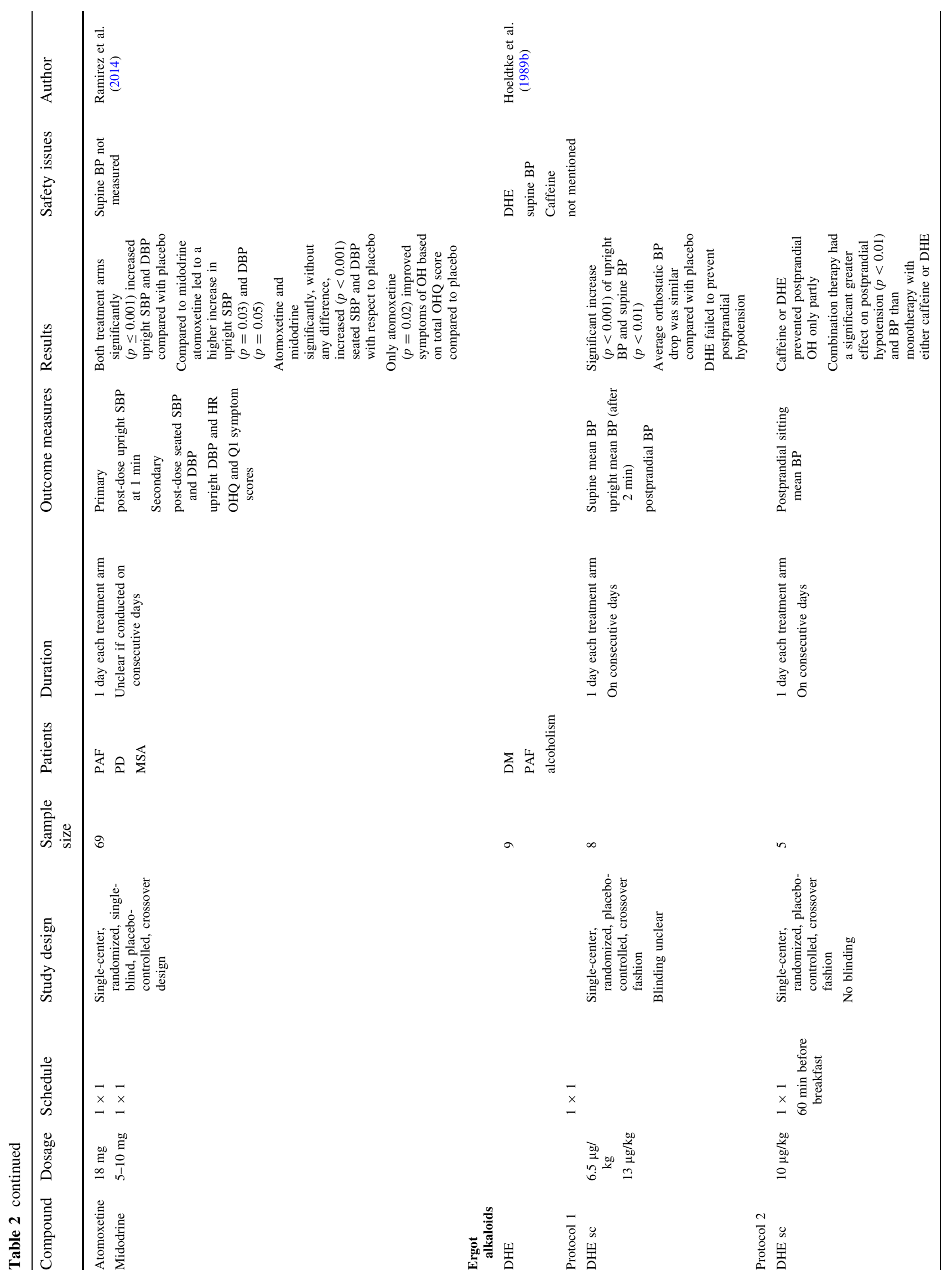




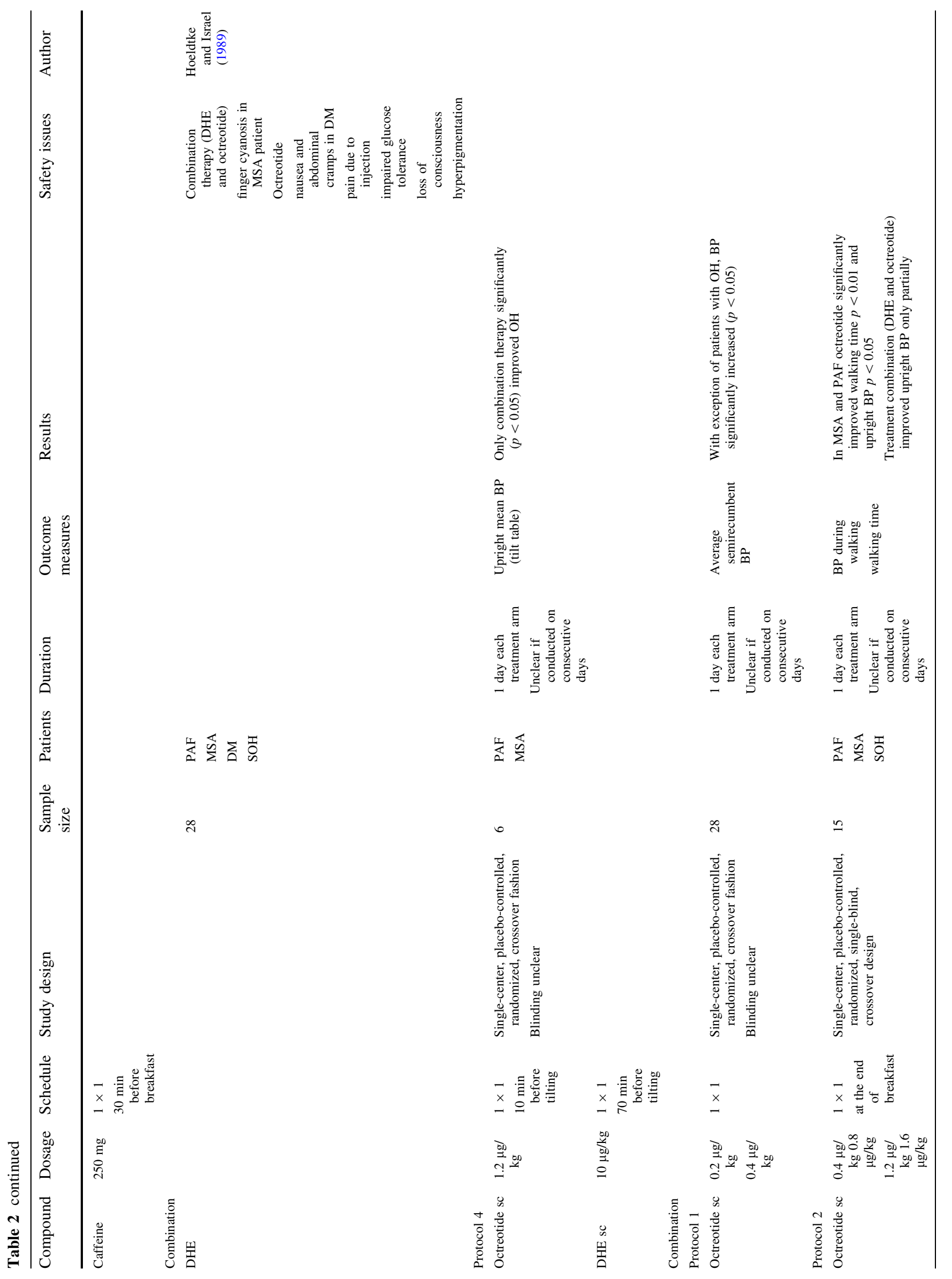




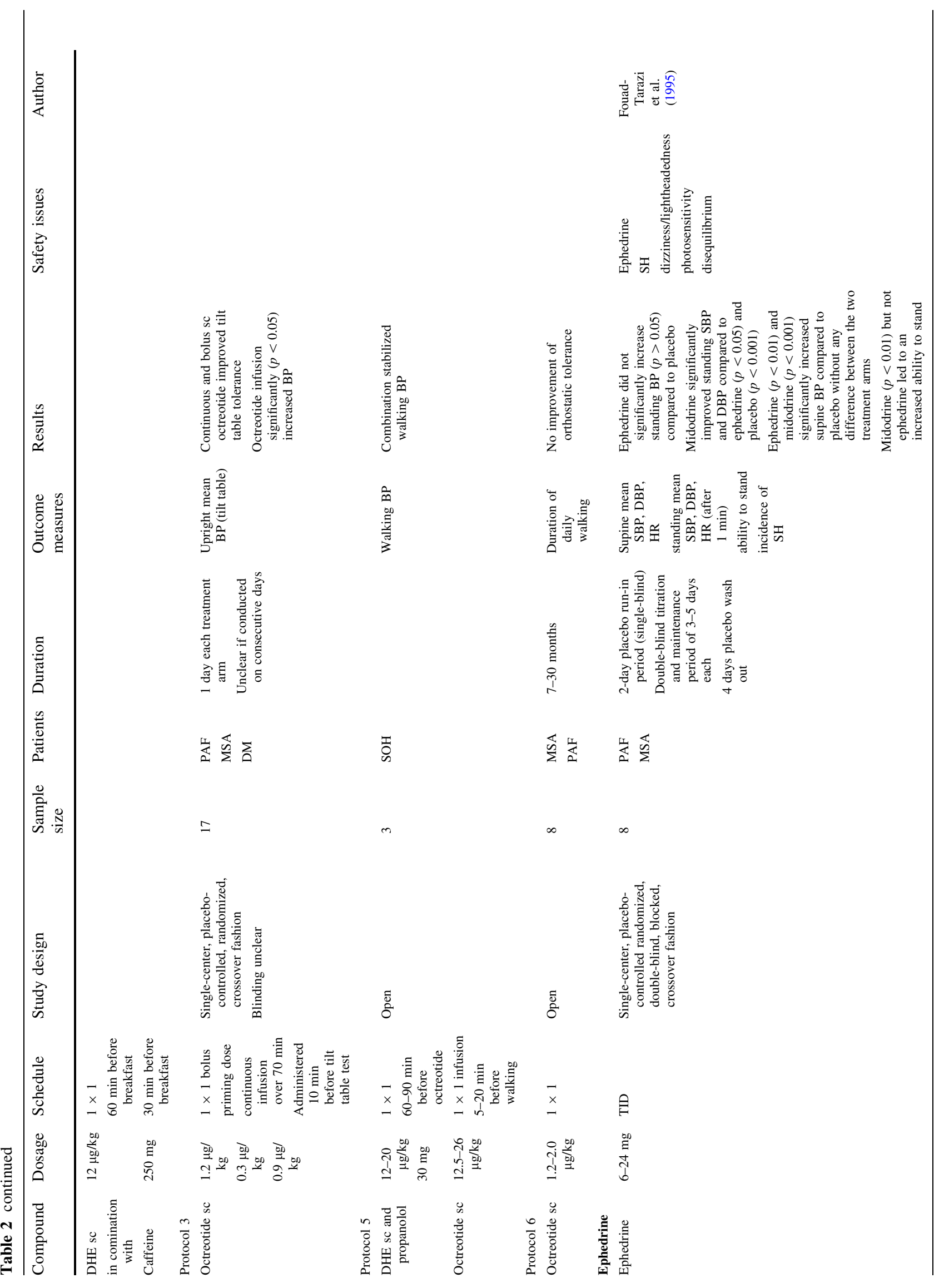




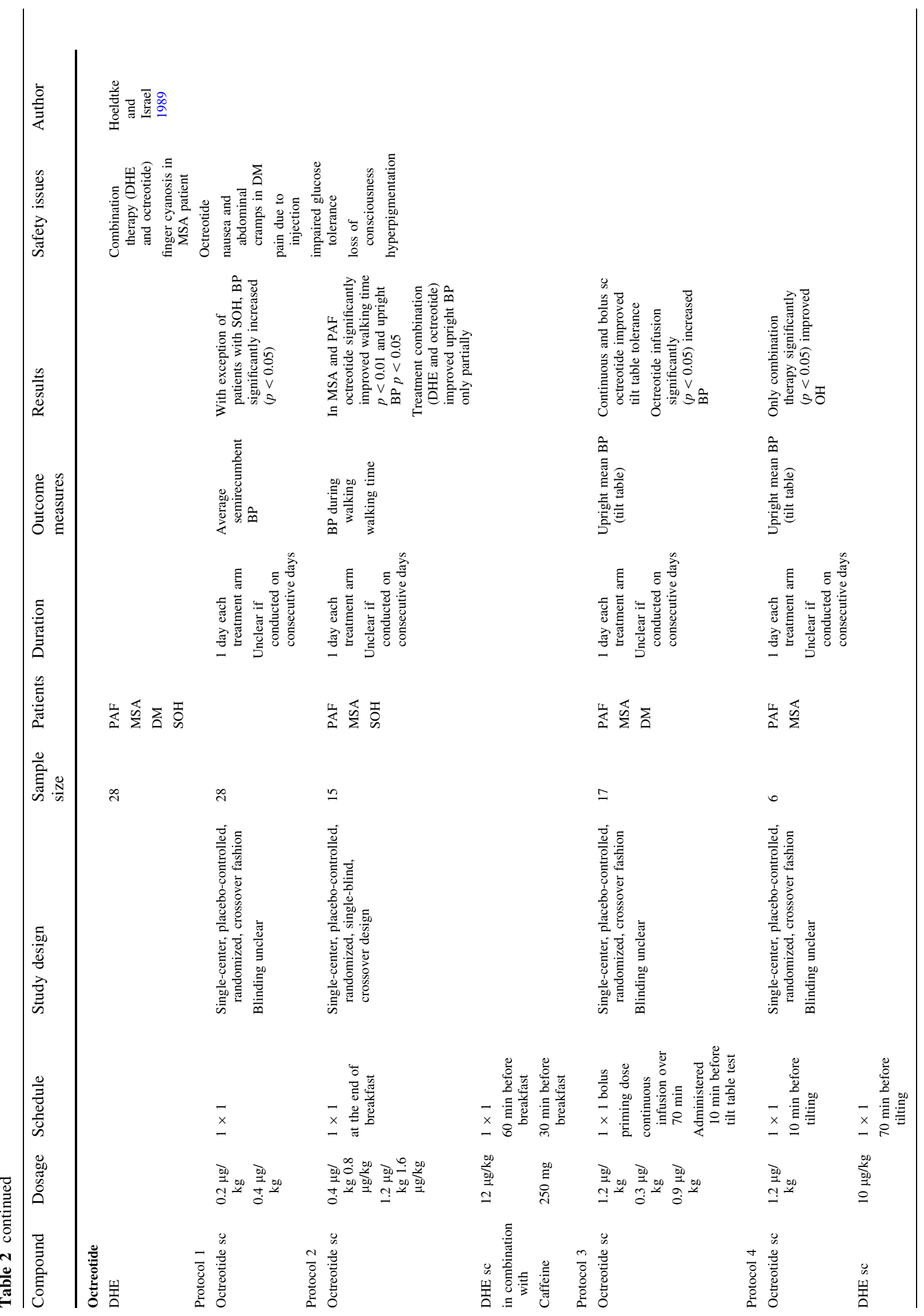




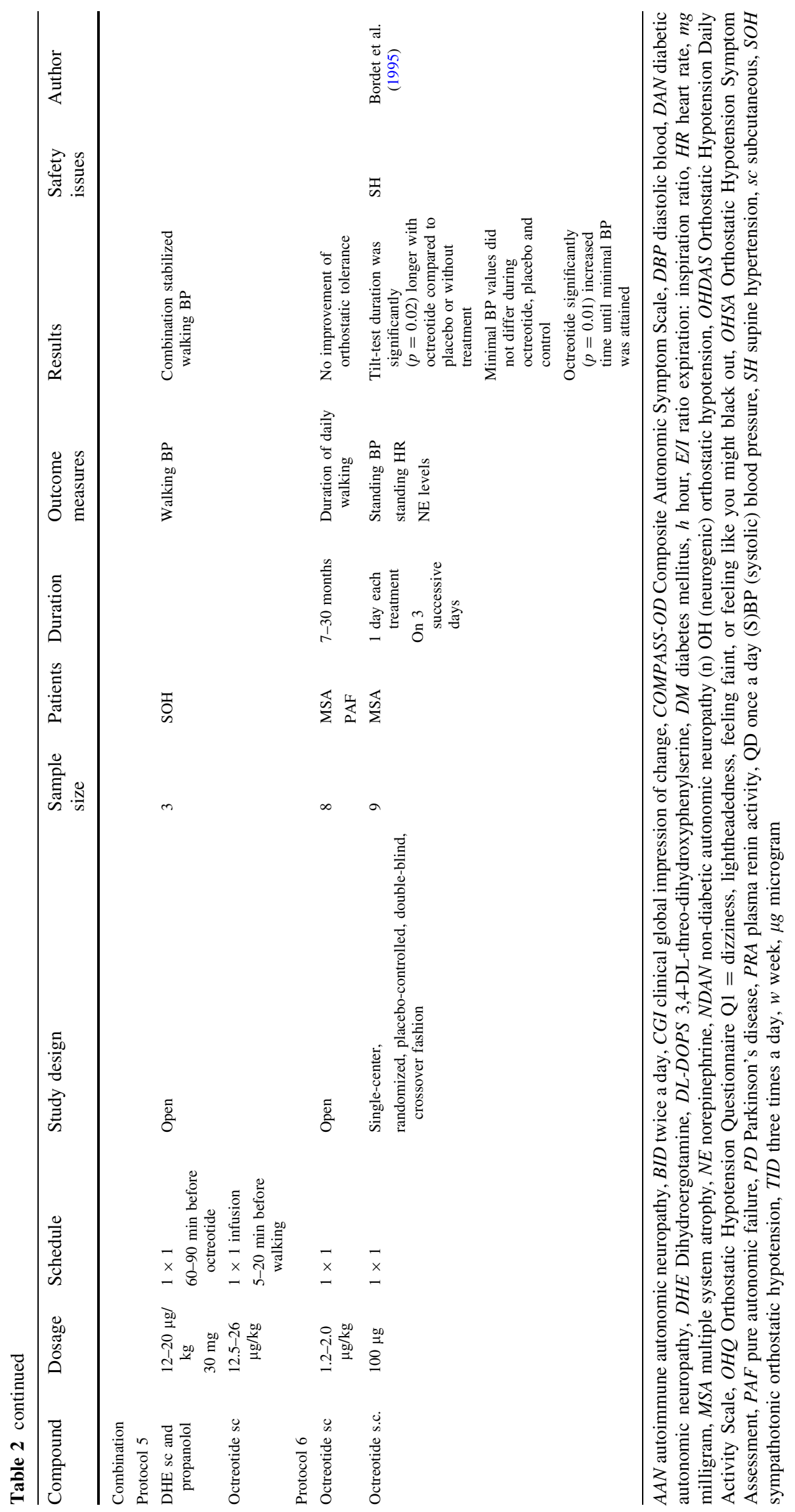




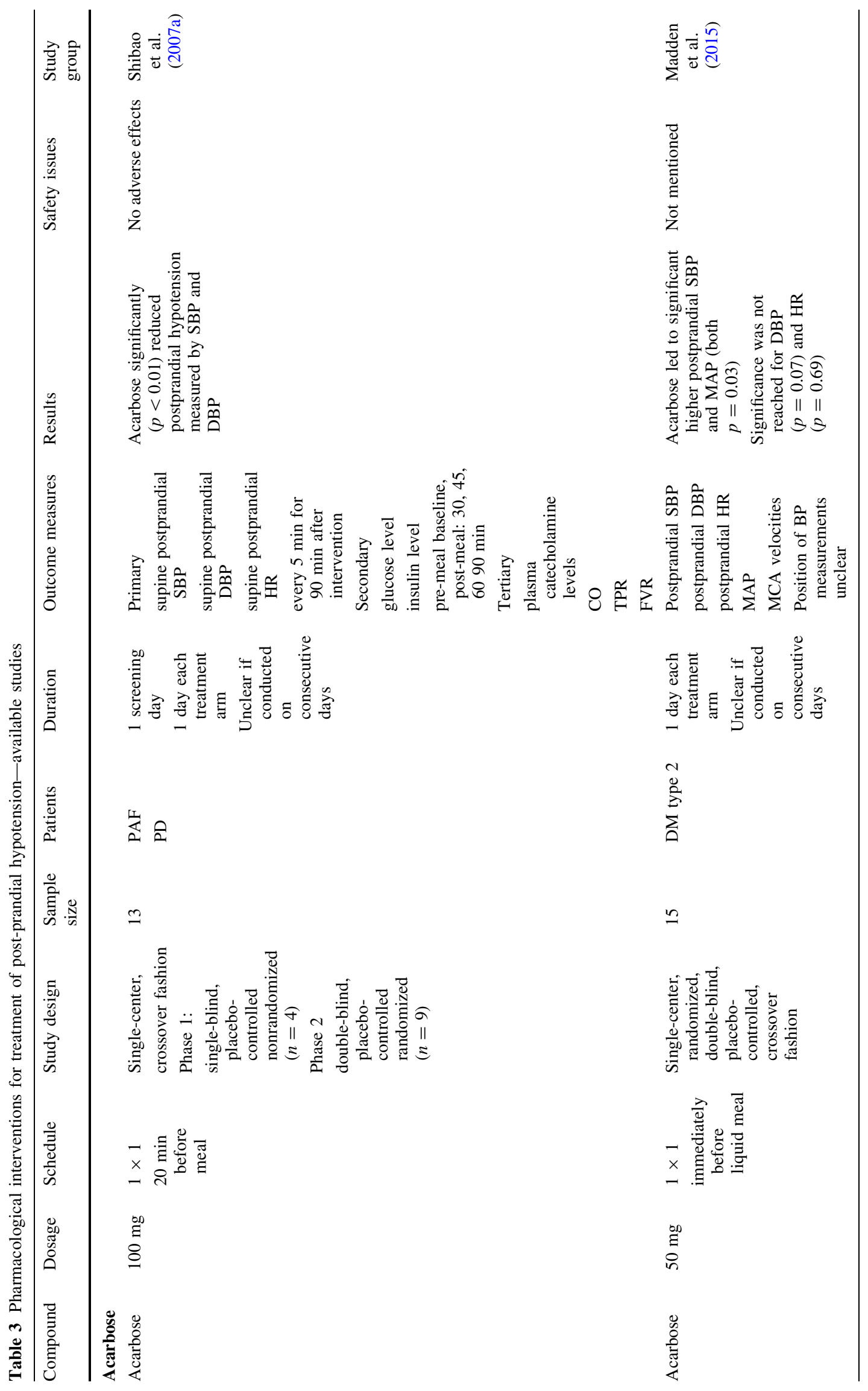




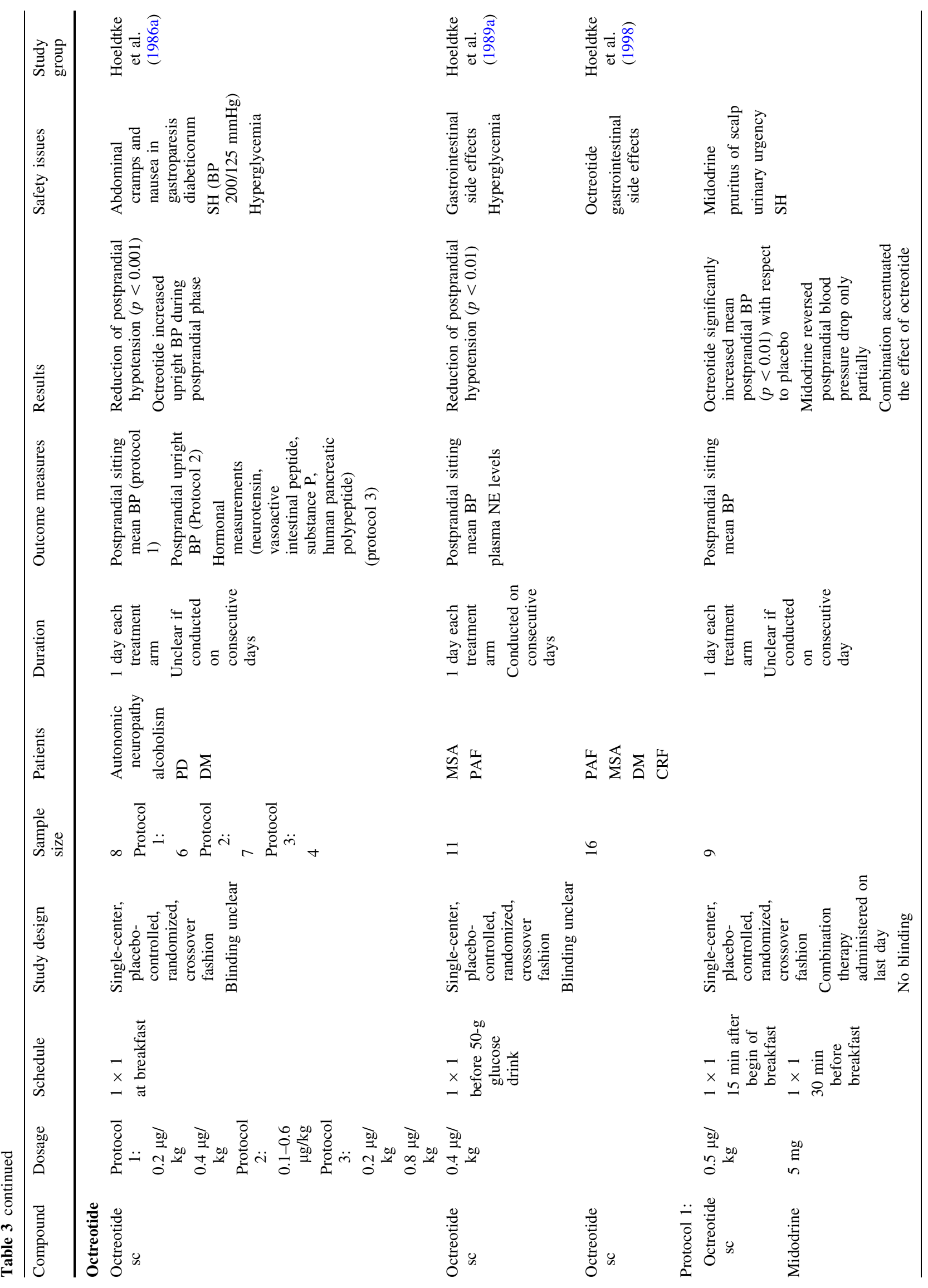




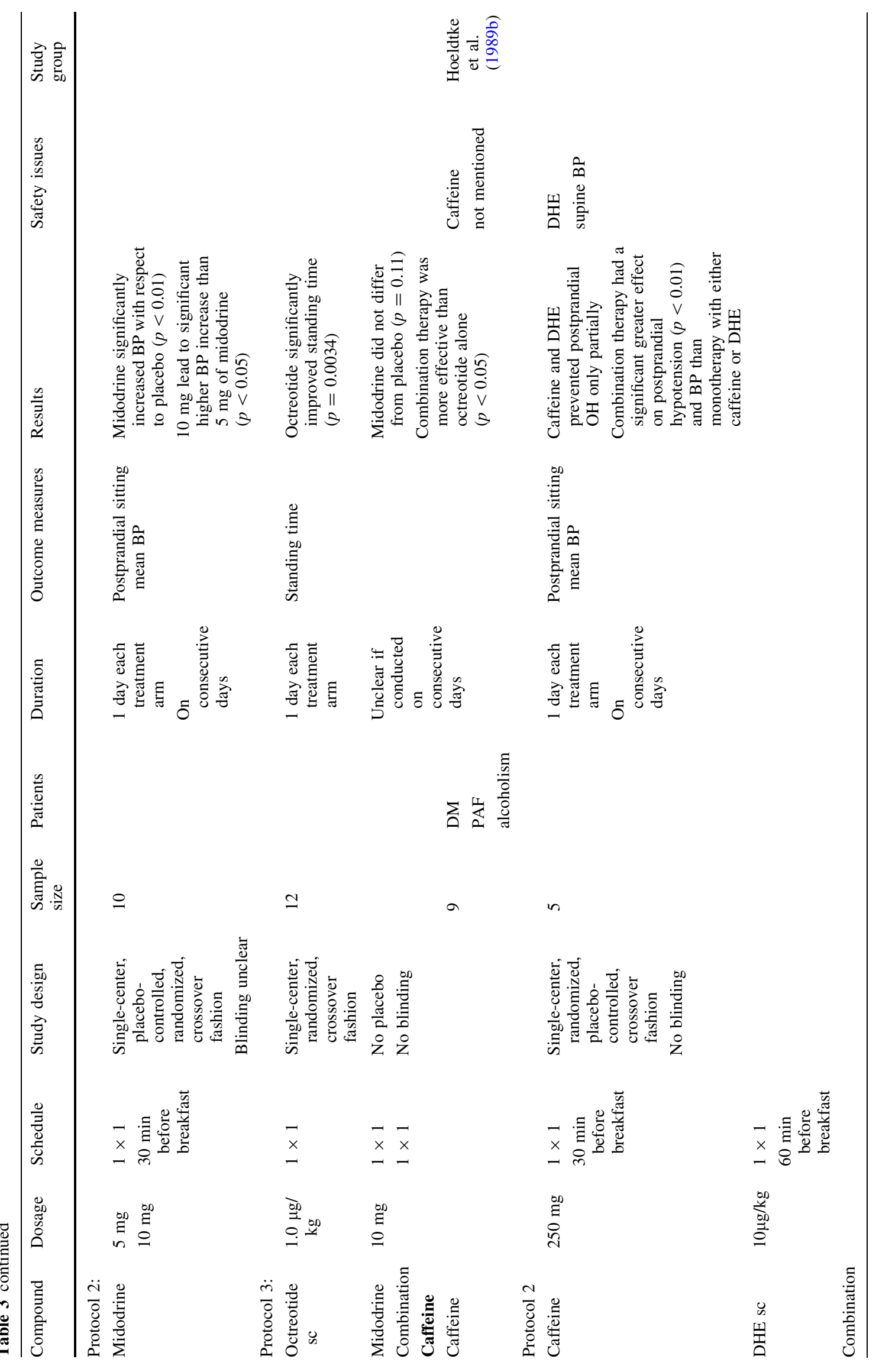




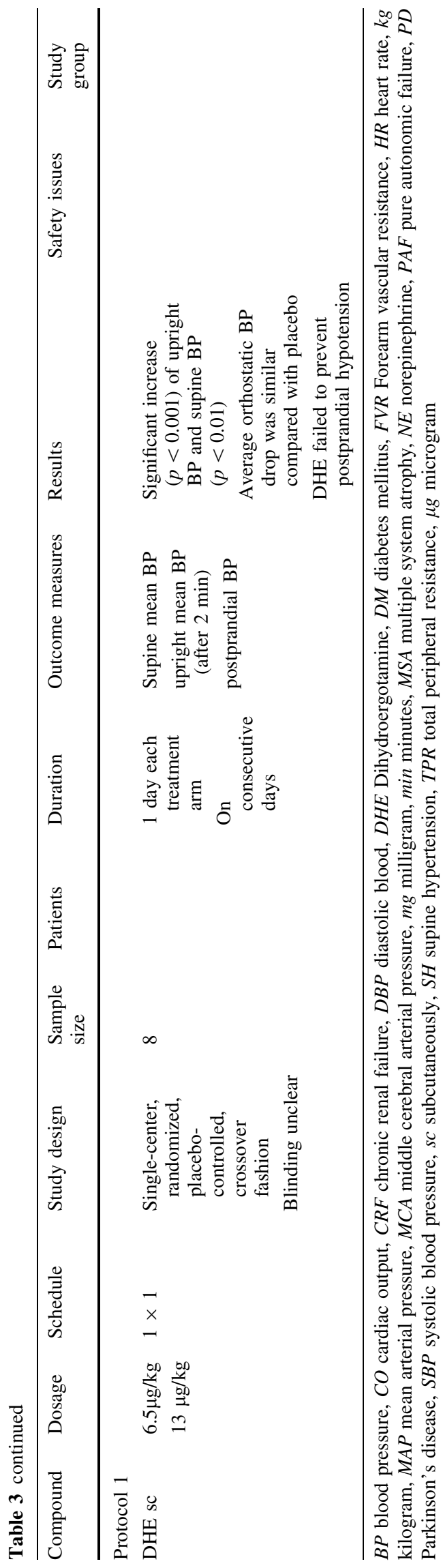

planned activities. Patients should be advised to stand up slowly and to perform physical countermaneuvers (leg crossing, squat position, bending forward) in case of dizziness. Sleeping in a head-up tilt position should be recommended to prevent supine hypertension and reduce nocturnal pressure natriuresis (Fanciulli et al. 2016b). Patients should be also educated to avoid trigger factors such as alcohol, heat exposure and large carbohydrate- or fat-rich meals and be aware that Valsalva-like maneuvers (e.g., micturition and defecation) may precipitate hypotensive episodes.

When aforementioned measures have been implemented and do not provide satisfactory relief, pharmacological therapy is required in addition. Pharmacological options are summarized in Table 6. As a general rule, drug choice should be tailored on the basis of expected benefit, relevant comorbidities, and potential adverse events. In younger, mobile patients, one may treat $\mathrm{nOH}$ more vigorously to warrant autonomy and symptoms' control in daily life. To the contrary, treatment of nOH may be less relevant than limiting polypharmacy in older, wheel-chair bound patients.

At present only midodrine and droxidopa (the latter in the US and Japan only) have been approved for treatment of $\mathrm{nOH}$, while the remaining substances are prescribed in an off-label regimen.

Based on available literature, the quality of evidence is high and recommendation level is strong for midodrine. Starting dose is $2.5 \mathrm{mg}$ two to three times a day, which can be increased up to $10 \mathrm{mg} 3$ times a day. Midodrine can be administered in mono- or combined therapy with plasma volume expanders, such as fludrocortisone (Lahrmann et al. 2006). Use of midodrine is contraindicated in patients with severe cardiac disease, renal failure and urinary retention (McClellan et al. 1998).

Quality of evidence is moderate for droxidopa and the level of recommendation strong. Droxidopa should be initiated with a dosage of $100 \mathrm{mg}$ three times a day and could be titrated up to a maximum dosage of $600 \mathrm{mg}$ three times a day (Ricci et al. 2015). Notably, long-term data on safety and efficacy of droxidopa are still missing, limiting its use as first line therapy in clinical practice.

Fludrocortisone (quality of evidence very low, weak recommendation strength) can be considered as secondline agent, either as monotherapy $(0.1-0.2 \mathrm{mg}$ daily) or in combination with midodrine, if supine hypertension has been excluded (Freeman 2008). Importantly, fludrocortisone is contraindicated in patients with heart failure, kidney failure and hypertension (Ricci et al. 2015) and electrolyte monitoring is recommended to rule out hypokalemia.

Atomoxetine (18 mg, single dose) showed promising results in recent studies and may be considered in selected 
Table 4 Quality of evidence (high, moderate, low, very low) and recommendations (strong, weak) for non-pharmacological and pharmacological treatment of $\mathrm{nOH}$ and post-prandial hypotension according to GRADE (Leone et al. 2013; https://gradepro.org)

\begin{tabular}{|c|c|c|c|}
\hline intervention & $\begin{array}{l}\text { quality of evidence of } \\
\text { reduction BP drop }\end{array}$ & recommendation & safety issues \\
\hline \multicolumn{4}{|c|}{ Non-pharmacological interventions } \\
\hline abdominal binder & moderate & strong & none \\
\hline sodium intake & low & weak & none \\
\hline water & very low & weak & none \\
\hline meal size & very low & weak & none \\
\hline nocturnal head-up tilt position & very low & weak & none \\
\hline physical counter maneuvers & very low & weak & none \\
\hline compression stockings & very low & weak & none \\
\hline \multicolumn{4}{|c|}{ Pharmacological interventions for $\mathrm{nOH}$} \\
\hline midodrine & high & strong & $\begin{array}{l}\text { supine hypertension, urinary retention, piloerection, } \\
\text { scalp pruritus, paresthesia, chills, headache }\end{array}$ \\
\hline droxidopa & moderate & strong & $\begin{array}{l}\text { supine hypertension, headache, dizziness, fatigue, } \\
\text { syncope, gastrointestinal complaints, urinary tract } \\
\text { symptoms }\end{array}$ \\
\hline atomoxetine & low & weak & $\begin{array}{c}\text { not mentioned } \\
\text { effect on supine BP unclear }\end{array}$ \\
\hline octreotide & low & weak & $\begin{array}{l}\text { gastrointestinal side effects especially in gastroparesis } \\
\text { diabetoricum, hyperglycemia, supine hypertension }\end{array}$ \\
\hline fludrocortisone & very low & weak & $\begin{array}{l}\text { supine hypertension, nausea, headache, } \\
\text { lightheadedness, dizziness, edema, hypokalemia, renal } \\
\text { and cardiac fibrosis (long-term), end organ damage } \\
\text { (long-term) }\end{array}$ \\
\hline pyridostigmine & very low & weak & gastrointestinal side effects, urinary urgency \\
\hline yohimbine & very low & weak & not mentioned, effect on supine BP unclear \\
\hline fluoxetine & very low & weak & $\begin{array}{c}\text { gastrointestinal symptoms, symptoms referable to } \\
\text { nervous system (e.g. headache, dizziness) }\end{array}$ \\
\hline $\begin{array}{l}\text { ergotamine } \\
\text { dihydroergotamine }\end{array}$ & very low & weak & $\begin{array}{c}\text { nausea, vomiting, paresthesias, fatigue, fibrosis } \\
\text { (retroperitoneal, cardiac, pleural, pulmonary), } \\
\text { peripheral vasoconstriction, ergotism }\end{array}$ \\
\hline erythropoietin & very low & weak & $\begin{array}{l}\text { flu-like symptoms, allergic reactions, hypertension, } \\
\text { increased risk of thrombosis }\end{array}$ \\
\hline ephedrine & very low & weak & $\begin{array}{c}\text { supine hypertension, dizziness, lightheadedness, } \\
\text { photosensitivity, disequilibrium }\end{array}$ \\
\hline phenylpropanolamine pseudoephedrine & very low & weak & $\begin{array}{c}\text { supine hypertension, central sympathomimetic adverse } \\
\text { events, cardiovascular events }\end{array}$ \\
\hline $\begin{array}{l}\text { indomethacin, ibuprofen, caffeine, } \\
\text { methylphenidate and desmopressin }\end{array}$ & very low & weak & 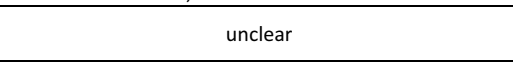 \\
\hline \multicolumn{4}{|c|}{ Pharmacological interventions for postprandial hypotension } \\
\hline acarbose & moderate & strong & $\begin{array}{l}\text { gastrointestinal side effects (e.g. flatulence, diarrhea, } \\
\text { stomachache) }\end{array}$ \\
\hline octreotide & moderate & strong & $\begin{array}{c}\text { gastrointestinal side effects especially in patients with } \\
\text { gastroparesis diabeticorum } \\
\text { hyperglycemia } \\
\text { supine hypertension }\end{array}$ \\
\hline voglibose & very low & weak & gastrointestinal side effects \\
\hline caffeine & very low & weak & not mentioned \\
\hline
\end{tabular}

patients with refractory $\mathrm{nOH}$, proven they have no cerebrovascular or cardiac comorbidities and are not on treatment with monoamine oxidase inhibitors. Data on longterm efficacy and safety are missing for atomoxetine, determining a low quality of evidence and a weak recommendation level.

Quality of evidence is very low and recommendation weak for ephedrine (25-50 mg three times a day) (Freeman 2008). Erythropoietin in combination with iron supplementation may have a positive impact on symptoms of
$\mathrm{nOH}$, especially in anemic patients, but risk of supine hypertension and polycythemia have to be considered (Kaufmann et al. 2015; Freeman 2008). For pyridostigmine, yohimbine, ergotamine, dihydroergotamine, ephedra alkaloids, desmopressin, indomethacin and fluoxetine the quality of evidence is very low, for octreotide low. Based on inconsistent results implications for clinical practice for all these treatment options remain investigational and therefore, recommendations are weak. Considering 
Table 5 Non-pharmacological management of nOH

Increase in salt (6-8 g/day) intake

Increase in fluid (1.5-2 1/day) intake

Bolus ingestion of $500 \mathrm{ml}$ water

Abdominal binders alone or with compression stockings

Physical countermaneuvers: leg crossing, squat position, bending forward

Sleeping with head-up tilt $30^{\circ}$

Avoidance of large fat- or carbohydrate-rich meals

Avoidance of trigger factors: alcohol, heat exposure, physical exertion

Awareness of Valsalva-like maneuvers (e.g., micturition, defecation)

Table 6 Pharmacological management of $\mathrm{nOH}$ and post-prandial hypotension

Orthostatic hypotension (CAVE: last medication $\geq 4 \mathrm{~h}$ before bedtime)

Midodrine 2.5-10 mg; TID

Fludrocortisone $0.1-0.2 \mathrm{mg}$; QD

Droxidopa $3 \times 100-600 \mathrm{mg}$; TID

Atomoxetine $18 \mathrm{mg}$; QD

Ephedrine 25-50 mg; TID

Erythropoietin in combination with iron supplementation

In selected cases: pyridostigmine, yohimbine, ergotamine, dihydroergotamine, ephedra alkaloids, desmopressin, indomethacin, fluoxetine

Post-prandial hypotension (administer before meal)

Acarbose $100 \mathrm{mg}$

Octreotide $1 \mu \mathrm{g} / \mathrm{kg}$ sc (contraindication: DM)

Caffeine $250 \mathrm{mg}$

$D M$ diabetes mellitus, $h$ hours, $k g$ kilogram, $m g$ milligram, $\mu g$ microgram, $Q D$ once a day, $T I D$ three times a day

individual risk-benefit ratio, abovementioned supplementary agents might be evaluated in selected cases.

It has to be emphasized that, although anti-hypotensive agents are frequently prescribed in combination in clinical practice, there is little evidence on this approach, especially regarding long-term benefits and safety.

Hypertensive BP fluctuations in the supine position are a common problem in patients with cardiovascular autonomic failure and limit management of $\mathrm{nOH}$. Patients taking any anti-hypotensive medication should be educated to avoid the recumbent position during daytime (Friedrich et al. 2010) and sleep with a head-up position overnight (Ten Harkel et al. 1992). All anti-hypotensive agents should not be taken $<4 \mathrm{~h}$ before bedtime and BP in the supine position should be monitored regularly.

In severe postprandial hypotension with limited response to conservative management (avoidance of large fat- or carbohydrate-rich meals and alcohol, increase in fluid and salt intake), acarbose in a dosage of $100 \mathrm{mg}$ can be used in selected cases (quality of evidence is moderate, recommendation strong). Alternatively, subcutaneous octreotide (quality of evidence moderate, recommendation strong, $1 \mu \mathrm{g} / \mathrm{kg}$ ) may be helpful (Wenning et al. 2005). Octreotide is contraindicated in patients with diabetes mellitus, given the risk of causing post-prandial hyperglycemia.

Although the quality of evidence is low and recommendation weak, caffeine in a dosage of $250 \mathrm{mg}$ may be considered as further alternative, given its favorable safety profile.

\section{Conclusion}

The target of nOH management is improvement of patient's quality of life by warranting mobility and preventing injurious falls due to syncope and pre-syncope. Although $\mathrm{nOH}$ has a high prevalence in the aging population, evidence-based data are limited. The mainstay of clinical management is, therefore, an individually tailored therapy, based on both non-pharmacological and pharmacological measures, which needs to be regularly evaluated and adapted over time, especially if the case of dynamic clinical scenarios, like in neurodegenerative diseases.

Acknowledgements Open access funding provided by the Medical University of Innsbruck.

Authors contributions SE: systematic review of the literature, writing of the first manuscript. GW: conception, critical revision of the manuscript. AF: conception, design, critical revision of the manuscript

\section{Compliance with ethical standards}

Conflict of interest Academic work, no external financial support allotted. The authors declare no conflicts of interest.

Open Access This article is distributed under the terms of the Creative Commons Attribution 4.0 International License (http://crea tivecommons.org/licenses/by/4.0/), which permits unrestricted use, distribution, and reproduction in any medium, provided you give appropriate credit to the original author(s) and the source, provide a link to the Creative Commons license, and indicate if changes were made.

\section{References}

Alam M, Smith G, Bleasdale-Barr K, Pavitt DV, Mathias CJ (1995) Effects of the peptide release inhibitor, octreotide, on daytime hypotension and on nocturnal hypertension in primary autonomic failure. J Hypertens 13(12 Pt 2):1664-1669

Armstrong E, Mathias CJ (1991) The effects of the somatostatin analogue, octreotide, on postural hypotension, before and after 
food ingestion, in primary autonomic failure. Clin Auton Res 19(1):135-140

Axelrod F, Krey L, Glickstein J, Allison JW, Friedman D (1995) Preliminary observations on the use of midodrine in treating orthostatic hypotension in familial dysautonomia. J Auton Nerv Syst 55(1-2):29-35

Axenti I, Petelenz T, Sosnoski M, Slomińska-Petelenz T (1993) Gutron treatment of patients suffering from essential orthostatic hypotony. Part one-hemodynamic non-invasive investigation. Int Angiol 12(2):132-137

Beirão I, Lobato L, Moreira L, Mp Costa P, Fonseca I, Cabrita A, Porto G (2008) Long-term treatment of anemia with recombinant human erythropoietin in familial amyloidosis TTR V30M. Amyloid 15(3):205-209

Biaggioni I (2014) New developments in the management of neurogenic orthostatic hypotension. Current Cardiology Reports 16(11):542

Biaggioni I, Robertson D (1987) Endogenous restoration of noradrenaline by precursor therapy in dopamine-beta-hydroxylase deficiency. Lancet 2(8569):1170-1172

Biaggioni I, Onrot J, Stewart CK, Robertson D (1987) The potent pressor effect of phenylpropanolamine in patients with autonomic impairment. JAMA 258(2):236-239

Biaggioni I, Zygmunt D, HaileV Robertson D (1990) Pressor effect of inhaled ergotamine in orthostatic hypotension. Am J Cardiol 65(1):89-92

Biaggioni I, Robertson RM, Robertson D (1994a) Manipulation of norepinephrine metabolism with yohimbine in the treatment of autonomic failure. J Clin Pharmacol 34(5):418-423

Biaggioni I, Robertson D, Krantz S, Jones M (1994b) The anemia of primary autonomic failure and its reversal with recombinant erythropoietin. Ann Intern Med 121(3):181-186

Biaggioni I, Freeman R, Mathias CJ, Low P, Hewitt LA, Kaufmann H (2015) Randomized withdrawal study of patients with symptomatic neurogenic orthostatic hypotension responsive to droxidopa. Hypertension 65(1):101-107

Bordet R, Benhadjali J, Libersa C, Destee A (1994) Octreotide in the management of orthostatic hypotension in multiple system atrophy: pilot trial of chronic administration. Clin Neuropharmacol 17(4):380-383

Bordet R, Benhadjali J, Destée A, Belabbas A, Libersa C (1995) Octreotide effects on orthostatic hypotension in patients with multiple system atrophy: a controlled study of acute administration. Clin Neuropharmacol 18(1):83-89

Bouvette C, McPhee B, Opfer-Gehrking T, Low P (1996) Role of physical countermaneuvers in the management of orthostatic hypotension : efficacy and biofeedback augmentation. Mayo Clinic Proc 71(9). Mayo Foundation for Medical Education and Research 847-53

Cariga P, Mathias CJ (2001) Haemodynamics of the pressor effect of oral water in human sympathetic denervation due to autonomic failure. Clin Sci 101(3):313-319

Cariga P, Ahmed S, Mathias CJ, Gardner B (2002) The prevalence and association of neck (coat-hanger) pain and orthostatic (postural) hypotension in human spinal cord injury. Spinal Cord 40(2):77-82

Carvalho MJ, Van Den Meiracker AH, Boomsma F, Man In'TVeld AJ, Freitas J, Costa O, Falcao De Freitas A (1997) Improved orthostatic tolerance in familial amyloidotic polyneuropathy with unnatural noradrenaline precursor L-Threo-3,4-dihydroxyphenylserine. J Auton Nerv Syst 62(1-2):63-71

Deguchi K, Ikeda K, Sasaki I, Shimamura M, Urai Y, Tsukaguchi M, Touge T, Takeuchi H, Kuriyama S (2007) Effects of daily water drinking on orthostatic and postprandial hypotension in patients with multiple system atrophy. J Neurol 254(6):735-740
Denq J, Opfer-Gehrking T, Giuliani M, Felten J, Convertino V, Low P (1997) Efficacy of compression of different capacitance beds in the amelioration of orthostatic hypotension. Clin Auton Res 7(6):321-326

Eagleton H, Littlewood T (2003) Update on the clinical use and misuse of erythropoietin. Curr Hematol Rep. 2(2):109-115

Elizondo L, Doerr D, Sims M, Hoffler G, Convertino V (1996) Application of USAF G-suit technology for clinical orthostatic hypotension: a case study. Aviat Space Environ Med 67(4):344-350

Fanciulli A, Wenning GK (2015) Multiple-system atrophy. N Engl J Med 372(3):249-263

Fanciulli A, Strano S, Colosimo C, Caltagirone C, Spalletta G, Pontieri FE (2013) The potential prognostic role of cardiovascular autonomic failure in $\alpha$-synucleinopathies. Eur $\mathbf{J}$ Neurol 20(2):231-235

Fanciulli A, Goebel G, Metzler B, Sprenger F, Poewe W, Wenning GK, Seppi K (2016a) Elastic abdominal binders attenuate orthostatic hypotension in Parkinson's disease. Mov Disord Clin Pract 3(2): 156-160

Fanciulli A, Göbel G, Ndayisaba JP, Granata R, Duerr S, Strano S, Colosimo C, Poewe W, Pontieri F, Wenning GK (2016b) Supine hypertension in Parkinson's disease and multiple system atrophy. Clin Auton Res 26(2):97-105

Figueroa J, Singer W, Sandroni P, Sletten D, Gehrking T, Gehrking J, Low P, Basford J (2015) Effects of patient-controlled abdominal compression on standing systolic blood pressure in adults with orthostatic hypotension. Arch Phys Med Rehabil 96(3):505-510

Fouad-Tarazi FM, Okabe M, Goren H (1995) Alpha sympathomimetic treatment of autonomic insufficiency with orthostatic hypotension. Am J Med 99(6):604-610

Freeman Roy (2008) Clinical practice. Neurogenic orthostatic hypotension. N Engl J Med 358(6):615-624

Freeman R, Landsberg L, Young J (1999) The treatment of neurogenic orthostatic hypotension with 3,4-DL-threo-dihydroxyphenylserine a randomized, placebo-controlled, crossover trial. Neurology 53(9):2151-2157

Freeman R, Wieling W, Axelrod F, Benditt D, Benarroch E, Biaggioni I, Cheshire WP et al (2011) Consensus statement on the definition of orthostatic hypotension, neurally mediated syncope and the postural tachycardia syndrome. Auton Neurosci Basic Clin 161(1-2):46-48

Friedrich C, Reichmann H, Ziemssen T (2010) Treatment of autonomic dysfunction in patients with extrapyramidal disorders. Fortschr Neurol Psychiatr 78(8):445-455

Frongillo D, Stocchi F, Buccolini P, Stecconi P, Viselli F, Ruggieri S, Cannata D (1995) Ambulatory blood pressure monitoring and cardiovascular function tests in multiple system atrophy. Fundam Clin Pharmacol 9(2):187-196

Fukushima T, Asahina M, Fujinuma Y, Yamanaka Y, Katagiri A, Mori M, Kuwabara S (2013) Role of intestinal peptides and the autonomic nervous system in postprandial hypotension in patients with multiple system atrophy. J Neurol 260(2):475-483

Goldstein D, Sharabi Y (2009) Neurogenic orthostatic hypotension: a pathophysiological approach. Circulation 119(1):139-146

Goldstein D, Holmes C, Sharabi Y, Wu T (2015) Survival in synucleinopathies: a prospective cohort study. Neurology 85(18):1554-1561

Grubb B, Samoil D, Kosinski D, Wolfe D, Lorton M, Madu E (1994) Fluoxetine hydrochloride for the treatment of severe refractory orthostatic hypotension. Am J Med 97(4):366-368

Ha A, Brown C, York M, Jankovic J (2011) The prevalence of symptomatic orthostatic hypotension in patients with Parkinson's disease and atypical parkinsonism. Parkinsonism Relat Disord 17(8):625-628 
Hakamäki T, Rajala T, Lehtonen A (1998) Ambulatory 24-hour blood pressure recordings in patients with Parkinson's disease with or without fludrocortisone. Int $\mathbf{J}$ Clin Pharmacol Ther 36(7):367-369

Hauser R, Hewitt L, Isaacson S (2014) Droxidopa in patients with neurogenic orthostatic hypotension associated with Parkinson's disease (NOH306A). J Parkinson's Dis 4(1):57-65

Hauser R, Isaacson S, Lisk J, Hewitt L, Rowse G (2015) Droxidopa for the short-term treatment of symptomatic neurogenic orthostatic hypotension in Parkinson's disease (nOH306B). Mov Disord 30(5):646-654

Hoeldtke R, Israel B (1989) Treatment of orthostatic hypotension with octreotide. J Clin Endocrinol Metab 68(6):1051-1059

Hoeldtke R, Streeten D (1993) Treatment of orthostatic hypotension with erythropoietin. N Engl J Med 329(9):611-615

Hoeldtke R, O'Dorisio T, Boden G (1986a) Treatment of autonomic neuropathy with a somatostatin analogue SMS-201-995. Lancet 2(8507):602-605

Hoeldtke R, Boden G, O'Dorisio T (1986b) Treatment of postprandial hypotension with a somatostatin analogue (SMS 201-995). Am J Med 81(6B):83-87

Hoeldtke R, Dworkin G, Gaspar S, Israel B, Boden G (1989a) Effect of the somatostatin analogue SMS-201-995 on the adrenergic response to glucose ingestion in patients with postprandial hypotension. Am J Med 86(6 Pt 1):673-677

Hoeldtke R, Cavanaugh S, Hughes J, Polansky M (1989b) Treatment of orthostatic hypotension with dihydroergotamine and caffeine. Ann Intern Med 105(2):168-173

Hoeldtke R, Horvath G, Bryner K, Hobbs G (1998) Treatment of orthostatic hypotension with midodrine and octreotide. J Clin Endocrinol Metab 83(2):339-343

Humm M, Mason L, Mathias C (2008) Effects of water drinking on cardiovascular responses to supine exercise and on orthostatic hypotension after exercise in pure autonomic failure. J Neurol Neurosurg Psychiatry 79(10):1160-1164

Illman A, Stiller K, Williams M (2000) The prevalence of orthostatic hypotension during physiotherapy treatment in patients with an acute spinal cord injury. Spinal Cord 38(12):741-747

Jankovic J, Gilden J, Hiner B, Kaufmann H, Brown D, Coghlan C, Rubin M, Fouad-Tarazi F (1993) Neurogenic orthostatic hypotension: a double-blind, placebo-controlled study with midodrine. Am J Med 95(1):38-48

Jordan J, Shannon J, Biaggioni I, Norman R, Black B, Robertson D (1998) Contrasting actions of pressor agents in severe autonomic failure. Am J Med 105(2):116-124

Jordan J, Shannon J, Grogan E, Biaggioni I, Robertson D (1999) A potent pressor response elicited by Drinking water. Lancet 353(9154):723

Jordan J, Shannon J, Black B, Ali Y, Farley M, Costa F, Diedrich A et al (2000) The pressor response to water drinking in humans: a sympathetic reflex? Circulation 101(5):504-509

Jordan J, Shannon J, Diedrich A, Black B, Robertson D, Biaggioni I (2004) Water potentiates the pressor effect of ephedra alkaloids. Circulation 109(15):1823-1825

Kachi T, Iwase S, Mano T, Saito M, Kunimoto M, Sobue I (1988) Effect of L-threo-3,4-dihydroxyphenylserine on muscle sympathetic nerve activities in Shy-Drager syndrome. Neurology 38(7):1091-1094

Kaufmann H (2016) Managment of cardiovascular autonomic failure. In 5th International Congress on Multiple System Atrophy, Salerno. (personal communication)

Kaufmann H, Brannan T, Krakoff L, Yahr M, Mandeli J (1988) Treatment of orthostatic hypotension due to autonomic failure with a peripheral alpha-adrenergic agonist (midodrine). Neurology 38(6):951-956
Kaufmann H, Oribe E, Yahr M (1991) Differential effect of L-threo3,4-dihydroxyphenylserine in pure autonomic failure and multiple system atrophy with autonomic failure. J Neural Transm 3(2):143-148

Kaufmann H, Saadia D, Voustianiouk A, Goldstein DS, Holmes S, Yahr M, Nardin R, Freeman R (2003) Norepinephrine precursor therapy in neurogenic orthostatic hypotension. Circulation 108(6):724-728

Kaufmann H, Freeman R, Low P, Pedder S, Hewitt L, Mauney J, Feirtag M, Mathias CJ (2014) Droxidopa for neurogenic orthostatic hypotension: a randomized, placebo-controlled, phase 3 trial. Neurology 83(4):328-335

Kaufmann H, Norcliffe-Kaufmann L, Palma J (2015) Droxidopa in neurogenic orthostatic hypotension. Expert Rev Cardiovasc Ther 13(8):875-891

Kawakami K, Abe H, Harayama N, Nakashima Y (2003) Successful treatment of severe orthostatic hypotension with erythropoietin. Pacing Clin Electrophysiol. 26(1 Pt 1):105-107

Köllensperger M, Geser F, Ndayisaba JP, Boesch S, Seppi K, Ostergaard K, Dupont E et al (2010) Presentation, diagnosis, and management of multiple system atrophy in Europe: final analysis of the European multiple system atrophy registry. Mov Disord 25(15):2604-2612

Lahrmann H, Cortelli P, Hilz M, Mathias CJ, Struhal W, Tassinari M (2006) EFNS guidelines on the diagnosis and management of orthostatic hypotension. Eur J Neurol 13(9):930-936

Leone M, Brainin M, Boon P, Pugliatti M, Keindl M, Bassetti C (2013) Guidance for the preparation of neurological management guidelines by EFNS scientific task forces-revised recommendations 2012. Eur Heart J 20(3):410-419

Lipp A, Tank J, Franke G, Arnold G, Luft F, Jordan J (2005) Osmosensitive mechanisms contribute to the water drinkinginduced pressor response in humans. Neurology 65(6):905-907

Low P (2008) Prevalence of orthostatic hypotension. Clin Auton Res 18(Suppl 1):8-13

Low P, Gilden J, Freeman R, Sheng K, McElligott M (1997) Efficacy of Midodrine vs Placebo in Neurogenic Orthostatic Hypotension. A randomized, double-blind multicenter study. JAMA 277(13):1046-1051

Low P, Benrud-Larson L, Sletten D, Opfer-Gehrking T, Weigand S, O'Brien P, Suarez G, Dyck P (2004) Autonomic symptoms and diabetic neuropathy: a population-based study. Diabetes Care 27(12):2942-2947

Madden K, Harris D, Meneilly G (2015) Attenuation of postprandial hypotension with acarbose in older adults with type 2 diabetes mellitus. J Am Geriatr Soc 63(7):1484-1486

Man in't Veld A, Boomsma F, van den Meiracker M, Schalekamp A (1987) Effect of unnatural noradrenaline precursor on sympathetic control and orthostatic hypotension in dopamine-betahydroxylase deficiency. Lancet 2(8569):1172-1175

Maruta T, Komai K, Takamori M, Yamada M (2006) Voglibose inhibits postprandial hypotension in neurologic disorders and elderly people. Neurology 66(9):1432-1434

Mathias C, Fosbraey P, da Costa D, Thornley A, Bannister R (1986) The effect of desmopressin on nocturnal polyuria, overnight weight loss, and morning postural hypotension in patients with autonomic failure. $\mathrm{Br}$ Med $\mathrm{J}$ (Clinical Research Ed.) 293(6543):353-354

Mathias CJ, da Costa D, Fosbraey P, Bannister R, Wood S, Bloom S, Christensen N (1989) Cardiovascular, biochemical and hormonal changes during food-induced hypotension in chronic autonomic failure. J Neurol Sci 94(1-3):255-269

Mathias C, Holly E, Armstrong E, Shareef M, Bannister R (1991) The influence of food on postural hypotension in three groups with chronic autonomic failure clinical and therapeutic implications. J Neurol Neurosurg Psychiatry 54(8):726-730 
Mathias C, Senard J, Braune S, Watson L, Aragishi A, Keeling J, Taylor M (2001) L-threo-dihydroxyphenylserine (L-threo-DOPS; Droxidopa) in the management of neurogenic orthostatic hypotension: a multi-national, multi-center, dose-ranging study in multiple system atrophy and pure autonomic failure. Clin Auton Res 11(4):235-242

Matsubara S, Sawa Y, Yokoji H, Takamori M (1990) Shy-drager syndrome. Effect of fludrocortisone and L-threo-3,4-dihydroxyphenylserine on the blood pressure and regional cerebral blood flow. J Neurol Neurosurg Psychiatry 53(11):994-997

Maule S, Tredici M, Dematteis A, Matteoda C, Chiandussi L (2004) Postprandial hypotension treated with acarbose in a patient with type 1 diabetes mellitus. Clin Auton Res 14(6):405-407

McClellan K, Wiseman L, Wilde M (1998) Midodrine. A review of its therapeutic use in the management of orthostatic hypotension. Drugs Aging 12(1):76-86

Montastruc J, Pelat M, Verwaerde P, Brefel-Courbon C, Tran M, Blin O, Rascol O, Senard J (1998) Fluoxetine in orthostatic hypotension of Parkinson's disease: a clinical and experimental pilot study. Fundam Clin Pharmacol 12(4):398-402

Norcliffe-Kaufmann L, Axelrod F, Kaufmann H (2013) Developmental abnormalities, blood pressure variability and renal disease in riley day syndrome. J Hum Hypertens 27(1):51-55

Okamoto L, Shibao C, Gamboa A, Choi L, Diedrich A, Raj S, Black B, Robertson D, Biaggioni I (2012) Synergistic effect of norepinephrine transporter blockade and alpha-2 antagonism on blood pressure in autonomic failure. Hypertension 59(3):650-656

Onrot J, Goldberg M, Biaggioni I, Hollister A, Kingaid D, Robertson D (1985) Hemodynamic and humoral effects of caffeine in autonomic failure. Therapeutic implications for postprandial hypotension. N Engl J Med 313(9):549-554

Onrot J, Goldberg M, Biaggioni I, Wiley R, Hollister A, Robertson D (1987) Oral yohimbine in human autonomic failure. Neurology 37(2):215-220

Perera R, Isola L, Kaufmann H (1995) Effect of recombinant erythropoietin on anemia and orthostatic hypotension in primary autonomic failure. Clin Auton Res 5(4):211-213

Perrin V (1985) Clinical pharmacokinetics of ergotamine in migraine and cluster headache. Clin Pharmacokinet 10(4):334-352

Pittner H, Stormann H, Enzenhofer R (1976) Pharmacodynamic actions of midodrine, a new alpha-adrenergic stimulating agent, and its main metabolite, ST 1059. Arzneimittelforschung 26(12):2145-2154

Puvi-Rajasingham S, Mathias CJ (1996) Effect of meal size on postprandial blood pressure and on postural hypotension in primary autonomic failure. Clin Auton Res 6(2):111-114

Raimbach S, Cortelli P, Kooner J, Bannister R, Bloom S, Mathias C (1989) Prevention of glucose-induced hypotension by the somatostatin analogue octreotide (SMS 201-995) in chronic autonomic failure: haemodynamic and hormonal changes. Clin Sci (Lond) 77(6):623-628

Raj S, Biaggioni I, Black B, Rali A, Jordan J, Taneja I, Harris P, Robertson D (2006) Sodium paradoxically reduces the gastropressor response in patients with orthostatic hypotension. Hypertension 48(2):329-334

Ramirez C, Okamoto L, Arnold A, Gamboa A, Diedrich A, Choi L, Raj S, Robertson D, Biaggioni I, Shibao C (2014) Efficacy of atomoxetine versus midodrine for the treatment of orthostatic hypotension in autonomic failure. Hypertension 64(6): 1235-1240

Rascol O, Perez-Lloret S, Damier P, Delval A, Derkinderen P, Destée A, Meissner W, Tison F, Negre-Pages L (2015) Falls in ambulatory non-demented patients with Parkinson's disease. J Neural Transm 122(10):1447-1455
Ricci F, De Caterina R, Fedorowski A (2015) Orthostatic hypotension: epidemiology, prognosis, and treatment. J Am Coll Cardiol 66(7):848-860

Sakakibara R, Matsuda S, Uchiyama T, Yoshiyama M, Yamanishi T, Hattori $T$ (2003) The effect of intranasal desmopressin on nocturnal waking in urination in multiple system atrophy patients with nocturnal polyuria. Clin Auton Res 13(2):106-108

Sakoda S, Suzuki T, Higa S, Ueji M, Kishimoto S, Matsumoto M, Yoneda S (1958) Treatment of orthostatic hypotension in shydrager syndrome with DL-threo-3,4-dihydroxyphenylserine: a case report. Eur Neurol 24(5):330-334

Sandroni P, Opfer-Gehrking T, Singer W, Low P (2005) Pyridostigmine for treatment of neurogenic orthostatic hypertension: a follow-up survey study. Clin Auton Res 15(1):51-53

Sasaki E, Goda K, Nagata K, Kitaoka H, Ohsawa N, Hanafusa T (2001) Acarbose improved severe postprandial hypotension in a patient with diabetes mellitus. J Diabetes Complicat 15(3): 158-161

Sauer J, Ring B, Witcher J (2005) Clinical pharmacokinetics of atomoxetine. Clin Pharmacokinet 44(6):571-590

Schiff P (2006) Ergot and its alkaloids. Am J Pharm Educ 70(5):98

Schoffer K, Henderson R, O’Maley K, O'Sullivan J (2007) Nonpharmacological treatment, fludrocortisone, and domperidone for orthostatic hypotension in Parkinson's disease. Mov Disord 22(11):1543-1549

Schrage W, Eisenach J, Dinenno F, Roberts S, Johnson C, Sandroni P, Low P, Joyner M (2004) Effects of midodrine on exerciseinduced hypotension and blood pressure recovery in autonomic failure. J Appl Physiol (Bethesda, MD: 1985) 97(5):1978-1984

Senard J, Chamontin B, Rascol A, Montastruc J (1992) Ambulatory blood pressure in patients with Parkinson's disease without and with orthostatic hypotension. Clin Auton Res 2(2):99-104

Senard J, Rascol O, Rascol A, Montastruc J (1993) Lack of yohimbine effect on ambulatory blood pressure recording: a double-blind cross-over trial in Parkinsonians with orthostatic hypotension. Fundam Clin Pharmacol 7(8):465-470

Senard J, Brefel C, Carel C, Tran M, Montastruc J (1999) Water drinking and the heart. Lancet 353(9168):1971-1972

Shannon J, Diedrich A, Biaggioni I, Tank J, Robertson R, Robertson D, Jordan J (2002) Water drinking as a treatment for orthostatic syndromes. Am J Med 112(15):355-360

Shibao C, Raj S, Gamboa A, Diedrich A, Choi L, Black B, Robertson D, Biaggioni I (2007a) Norepinephrine transporter blockade with atomoxetine induces hypertension in patients with impaired autonomic function. Hypertension 50(1):47-53

Shibao C, Gamboa A, Diedrich A, Dossett C, Choi L, Farley G, Biaggioni I (2007b) Acarbose, an alpha-glucosidase inhibitor, Attenuates postprandial hypotension in autonomic failure. Hypertension 50(1):54-61

Shibao C, Okamoto L, Gamboa A, Yu C, Diedrich A, Raj S, Robertson D, Biaggioni I (2010) Comparative efficacy of yohimbine against pyridostigmine for the treatment of orthostatic hypotension in autonomic failure. Hypertension 56(5):847-851

Siminoski K, Lukinuk C, Armstrong P (1988) Treatment of postural hypotension with aerosol ergotamine. CMAJ 139(1):51-52

Singer W, Opfer-Gehrking T, McPhee B, Hilz M, Bharucha A, Low P (2003) Acetylcholinesterase inhibition: a novel approach in the treatment of neurogenic orthostatic hypotension. Neurol Neurosurg Psychiatry 74(9):1294-1298

Singer W, Opfer-Gehrking T, Nickander K, Hines S, Low P (2006a) Acetylcholinesterase inhibition in patients with orthostatic intolerance. J Clin Neurophysiol 23(5):476-481

Singer W, Sandroni P, Opfer-Gehrking T, Suarez G, Klein C, Hines S, O'Brien P, Slezak J, Low P (2006b) Pyridostigmine treatment 
trial in neurogenic orthostatic hypotension. Arch Neurol 63(4):513-518

Smit A, Wieling W, Fujimura J, Denq J, Opfer-Gehrking T, Akarriou M, Karemaker J, Low P (2004) Use of lower abdominal compression to combat orthostatic hypotension in patients with autonomic dysfunction. Clin Auton Res 14(3):167-175

Smith G, Alam M, Watson L, Mathias C (1995) Effect of the somatostatin analogue, octreotide, on exercise-induced hypotension in human subjects with chronic sympathetic failure. Clin Sci (Lond) 89(4):367-373

Ten Harkel A, Van Lieshout J, Wieling W (1992) Treatment of orthostatic hypotension with sleeping in the head-up tilt position, alone and in combination with fludrocortisone. J Intern Med 232(2):139-145

Ten Harkel A, van Lieshout J, Wieling W (1994) Effects of leg muscle pumping and tensing on orthostatic arterial pressure: a study in normal subjects and patients with autonomic failure. Clin Sci (London, England: 1979) 87(5):553-558

Tilvis R, Hakala S, Valvanne J, Erkinjuntti T (1996) Postural hypotension and dizziness in a general aged population: a fouryear follow-up of the Helsinki aging study. J Am Geriatr Soc 44(7):806-814

Tutaj M, Marthol H, Berlin D, Brown C, Axelrod F, Hilz M (2006) Effect of physical countermaneuvers on orthostatic hypotension in familial dysautonomia. J Neurol 253(1):65-72

Van de Laar FA, Lucassen PL, Akkermans RP, Van de Lisdonk EH, Rutten GE, Van Weel C (2005) Alpha-glucosidase inhibitors for type 2 diabetes mellitus. Cochrane Database Syst Rev 18(2):CD003639

van Lieshout J, Ten Harkel A, van Leeuwen A, Wieling W (1991) Contrasting effects of acute and chronic volume expansion on orthostatic blood pressure control in a patient with autonomic circulatory failure. Neth J Med 39(1-2):72-83

van Lieshout J, ten Harkel A, Wieling W (1992) Physical manoeuvres for combating orthostatic dizziness in autonomic failure. Lancet 339(8798):897-898

van Lieshout J, Ten Harkel A, Wieling W (1999) Fludrocortisone and sleeping in the head-up position limit the postural decrease in cardiac output in autonomic failure. Clin Auton Res 10(1):35-42
Velseboer D, de Haan R, Wieling W, Goldstein D, de Bie R (2011) Prevalence of orthostatic hypotension in Parkinson's disease: a systematic review and meta-analysis. Parkinsonism Relat Disord 17(10):724-729

Victor R, Talman W (2002) Comparative effects of clonidine and dihydroergotamine on venomotor tone and orthostatic tolerance in patients with severe hypoadrenergic orthostatic hypotension. Am J Med 112(5):361-368

Wang A, Fealey R, Gehrking T, Low P (2008) Patterns of neuropathy and autonomic failure in patients with amyloidosis. Mayo Clin Proc 83(11):1226-1230

Wenning GK, Geser F, Poewe W (2005) Therapeutic strategies in multiple system atrophy. Mov Disord 20(Suppl 12):67-76

Wernicke JF (2004) Safety and side effect profile of fluoxetine. Expert Opin Drug Saf 3(5):495-504

Wieling W, van Lieshout J, van Leeuwen A (1993) Physical manoeuvres that reduce postural hypotension in autonomic failure. Clin Auton Res 3(1):57-65

Winkler A, Landau S, Watkins P (2001) Erythropoietin treatment of postural hypotension in anemic type 1 diabetic patients with autonomic neuropathy. Diabetes Care 24(6):1121-1123

Wright R, Kaufmann H, Perera R, Opfer-Gehrking T, McElligott M, Sheng K, Low P (1998) A double-blind, dose-response study of Midodrine in neurogenic orthostatic hypotension. Neurology 51(1):120-124

Yamamoto N, Sasaki E, Arishima T, Ito M, Tanaka H, Terasaki J, Imagawa A, Hanafusa $\mathrm{T}$ (2006) Combination therapy for postprandial and orthostatic hypotension in an elderly patient with type 2 diabetes mellitus. J Am Geriatr Soc 54(4):727-728

Young T, Mathias CJ (2004) The effects of water Ingestion on orthostatic hypotension in two groups of chronic autonomic failure: multiple system atrophy and pure autonomic failure. J Neurol Neurosurg Psychiatry 75(12):1737-1741

Z'Graggen W, Hess C, Humm A (2010) Acute fluid ingestion in the treatment of orthostatic intolerance-important implications for daily practice. Eur J Neurol 17(11):1370-1376 